\title{
Can We Use Ginkgo biloba Extract to Treat Alzheimer's Disease? Lessons from Preclinical and Clinical Studies
}

\author{
Liming Xie, Qi Zhu and Jiahong Lu *(D) \\ State Key Laboratory of Quality Research in Chinese Medicine, Institute of Chinese Medical Sciences, \\ University of Macau, Macau SAR 999078, China; mb95810@umac.mo (L.X.); yb67536@um.edu.mo (Q.Z.) \\ * Correspondence: jiahonglu@um.edu.mo; Tel.: +853-8822-4508
}

check for updates

Citation: Xie, L.; Zhu, Q.; Lu, J. Can We Use Ginkgo biloba Extract to Treat Alzheimer's Disease? Lessons from Preclinical and Clinical Studies. Cells 2022, 11, 479. https://doi.org/

10.3390/cells11030479

Academic Editors: Maria Antonietta

Panaro and Gérard Lizard

Received: 28 November 2021

Accepted: 24 January 2022

Published: 29 January 2022

Publisher's Note: MDPI stays neutral with regard to jurisdictional claims in published maps and institutional affiliations.

Copyright: (c) 2022 by the authors. Licensee MDPI, Basel, Switzerland. This article is an open access article distributed under the terms and conditions of the Creative Commons Attribution (CC BY) license (https:// creativecommons.org/licenses/by/ $4.0 /)$.

\begin{abstract}
Background: Ginkgo biloba extract (GBE) has been widely used to treat central nervous system and cardiovascular diseases. Accumulating evidence has revealed the therapeutic potential of GBE against Alzheimer's disease (AD); however, no systematic evaluation has been performed; (2) Methods: a total of 17 preclinical studies and 20 clinical trials assessing the therapeutic effects of GBE against AD were identified from electronic databases. The data in the reports were extracted to conduct a meta-analysis of the AD-related pathological features or symptoms; (3) Results: For the preclinical reports, 45 animals treated with GBE, in six studies, were subjected to cognitive function assessments by the Morris water maze. GBE was shown to reduce the escape latencies in several studies, in both rats and mice $\left(\mathrm{I}^{2}>70 \%, p<0.005\right)$. For the clinical trials, eight trials, including 2100 individuals, were conducted. The results show that GBE improved the SKT and ADAS-Cog scores in early-stage AD patients after high doses and long-term administration; (4) Conclusions: GBE displayed generally consistent anti-AD effects in animal experiments, and it might improve $\mathrm{AD}$ symptoms in early-stage AD patients after high doses and long-term administration. A lack of sample size calculations and the poor quality of the methods are two obvious limitations of the studies. Nevertheless, the preclinical and clinical data suggest that further large-scale clinical trials may be needed in order to examine the effects of long-term GEB administration on early-stage AD.
\end{abstract}

Keywords: Ginkgo biloba extract; Alzheimer's disease; clinical trial; meta-analysis

\section{Introduction}

Alzheimer's disease (AD) is an irreversible, age-related, and progressive neurodegenerative disease [1]. In 2019, Alzheimer's Disease International (ADI) estimated that more than 50 million people suffer from dementia worldwide, and they predict that the number will rise to 152 million by 2050, which would place an increasingly severe burden on societies and national economies [2]. At the same time, there are no known effective treatments for AD. Finding an effective therapy for AD would help millions of people, both directly, by improving their health, and indirectly, by relieving the burden on healthcare systems.

In terms of pathogenesis, amyloid plaques and neurofibrillary tangles are the two major structural changes in AD brains [3]. Currently, the amyloid hypothesis and the tau hypothesis are the generally accepted theories used to explain the etiology of AD. Amyloid- $\beta(A \beta)$ is the amyloid- $\beta$ precursor protein $(A \beta P P)$ cleavage product of a membrane protease called "secretase", and A $\beta$ aggregation is the major component of amyloid plaques in AD patients. Hyperphosphorylated tau aggregation is another major reason for the neurofibrillary tangles in AD. At present, the drugs on the market only alleviate the clinical symptoms, and cannot reverse the pathological changes and disease course of $\mathrm{AD}[4,5]$. On the basis of the current research, there is an urgent need to seek out a disease-modifying agent to slow down the progression of AD.

Ginkgo biloba is a famous herbal medicine that has been used in China since ancient times. In the 1970s, a standardized ginkgo biloba leaf extract (GBE), containing multiple 
pharmacologically active substances, was developed by Dr. Willmar Schwabe (Karlsruhe, Germany) [6]. It is a dry extract from G. biloba leaves (35-67:1), and the extraction solvent is acetone $(60 \% w / w)$. The extract contains $22.0-27.0 \%$ ginkgo flavonoids, including quercetin, kaempferol, and isorhamnetin [7], 5.0-7.0\% terpene lactones, consisting of 2.8-3.4\% ginkgolides A, B, and C and 2.6-3.2\% bilobalide, and ginkgolic acids at levels less than 5 PPM [8]. The main ingredients of standardized gingko biloba extract are flavonoids and terpenoids $[9,10]$. These components may be responsible for GBE's effects in the treatment of AD, which include: antioxidation, anti-inflammation, and antiapoptosis; protection against mitochondrial dysfunction, amyloidogenesis, and $\mathrm{A} \beta$ aggregation; the modulation of the ion homeostasis and phosphorylation of the tau protein; and even the induction of growth factors [11]. Several studies report that GBE significantly improved the performances of AD mice in the Morris water maze test [12]. Since 1985, a number of clinical trials have been conducted to evaluate the anti-AD efficacy of GBE. However, limited sample sizes and methodology flaws cast doubt on the value, accuracy, and reliability of these results. A systematic review is urgently needed to clarify what science has actually learned about the efficacy of GBE in treating AD.

This review reports the results of our systematic meta-analysis of articles on GBE for AD treatment in preclinical and clinical studies. In addition, we summarize the potential neuroprotective mechanisms of GBE in AD, determined from animal models. More importantly, we analyze and summarize the possible causes of the inconsistencies by comparing the effective and ineffective clinical trials. This review also provides a reference for the assessment of the methodological quality of AD preclinical and clinical studies.

\section{Materials and Methods}

\subsection{Search Strategy}

First, to identify articles focusing on the efficacy of Gingko biloba extract on AD animal models, a careful search was performed of the literature published between 2000 and 2020 and found in the electronic databases, Web of Science and PubMed. The key words used for the article search were ("Alzheimer") AND ("Ginkgo Biloba"). We also conducted a search with Google Scholar and included the first 200 hits, sorted by relevance [13]. Two individual reviewers (Liming Xie and Qi Zhu) worked independently to screen the abstracts of the qualified articles on the basis of the inclusion criteria (Table 1). When we disagreed, the article was screened by a third reviewer (Erjin Wang). Similarly, the clinical trial results were searched with three key words, ("Alzheimer disease") OR ("Dementia") AND ("Gingko biloba"), in the ClinicalTrials.gov website, and were searched with four key words, ("clinical trial"), ("Alzheimer disease") OR ("Dementia") AND ("Gingko biloba"), in Google Scholar.

Table 1. Inclusion and exclusion criteria for selecting preclinical articles.

\begin{tabular}{l}
\hline Inclusion Criteria: \\
\hline 1. Parallel experiments were conducted to evaluate the effects of EGB761 on AD protection \\
in vivo. \\
2. Laboratory animals of any species, age, sex, or strain to induce AD models were included. \\
3. Any kind of EGB761 intervention compared with a control group was included. Dosages, \\
methods of treatment, and curative times were not limited. \\
\hline Exclusion Criteria: \\
\hline Duplicated references; articles with incorrect and incomplete data; no access to the databases; \\
review articles, comments, letters, and case reports.
\end{tabular}

\subsection{Selection Criteria}

The inclusion and exclusion criteria for the selection of preclinical trials are listed in Table 1, and the criteria for the selection of clinical trials are presented in Table 2. 
Table 2. Inclusion and exclusion criteria for selecting clinical articles.

Inclusion Criteria:
1. The clinical trials were designed as double-blind randomized placebo-controlled trials.
2. The patients, by age, sex, administration route and duration, dosage, were included in the trials.
3. Specific and reliable criteria for the AD assessment, such as the SKT and MMSE, were included.
Exclusion Criteria:
Duplicated references; repetitive clinical data; articles with incorrect and incomplete data; no
access to the databases; review articles, comments, letters, and case reports.

\subsection{Data Extraction and Analysis}

After finishing the screening, two individual researchers extracted and tabulated the data from the selected articles. The details of the preclinical articles are listed in Table 3, with the following items: (1) First author name and publication year; (2) AD animal model; (3) Strains, weights, and sex of animals used; (4) Treatment dosage, duration, and administration route; and (5) Methods used in the experiments, with results corresponding to the methods. Specific information for the clinical study articles is listed in Table 4, with the following items: (1) Study, author(s), publication date; (2) Country; (3) Inclusion criteria; (4) Setting of study; (5) Duration; (6) Treatment; (7) Groups; (8) Age of participants; (9) Baseline scale; and (10) Withdrawal rate. Image J software was used to extract the numerical values from the graphs.

A meta-analysis was conducted with Review Manager 5.3 software. We processed the data with a fixed effects model and judged the heterogeneity with a $Q$ test and $\mathrm{I}^{2}$ statistics. Heterogeneity was considered to exist when $p<0.05$ : $\mathrm{I}^{2}=0 \%$ means no heterogeneity; $0<\mathrm{I}^{2} \leq 25 \%$ means mild heterogeneity; $25 \%<\mathrm{I}^{2} \leq 75 \%$ means moderate heterogeneity; and $\mathrm{I}^{2}>75 \%$ means a high degree of heterogeneity.

\subsection{Quality Assessment}

We scored the methodological quality of the included articles using the CAMARADES (Collaborative Approach to Meta-Analysis and Review of Animal Data from Experimental Stroke) list. Additionally, the preclinical study evaluation criteria were established in accordance with the characteristics of Alzheimer's disease. The criteria were as follows: (1) Publication in a peer-reviewed journal; (2) Random allocation of animals; (3) Outcomes assessed blindly; (4) Dose-response relationship assessed; (5) Appropriate animal model; (6) Necessary sample size calculation; (7) Observation of animal welfare regulations; and (8) No potential conflicts of interests [49]. Each article was given a quality score out of a maximum total of eight points. The clinical studies were evaluated similarly, with the addition of one more criterion: an ITT analysis (intent-to-treat analysis). 
Table 3. Characteristics of included animal studies related to GBE.

\begin{tabular}{|c|c|c|c|c|c|}
\hline Author & Animal Model & Treatment & Method & Result & Mechanism of Action \\
\hline $\begin{array}{l}\text { Ward, C. P. et al. } \\
\text { (2002) [12] }\end{array}$ & $\begin{array}{l}\text { C57BL/ } 6 \text { mice, male, } \\
20 \text { months old }\end{array}$ & $\begin{array}{l}\mathrm{EGb} 761(100 \mathrm{mg} / \mathrm{kg} / \text { day }) \text {, } \\
\text { orally for } 82 \text { consecutive days }\end{array}$ & $\begin{array}{l}\text { 1. Morris water maze test } \\
\text { 2. Elevated plus-maze test } \\
\text { 3. Protein levels of CREB }\end{array}$ & $\begin{array}{l}\uparrow \text { the time of hidden platform } \\
\downarrow \text { platform crossings (probe } \\
\text { test) } \\
\downarrow \text { time on the open arms } \\
\text { no significant differences }\end{array}$ & $\begin{array}{l}\text { Improved the learning and } \\
\text { memory cognition } \\
\text { Antioxidant properties }\end{array}$ \\
\hline $\begin{array}{l}\text { Stackman, R. W. et al. } \\
\text { (2003) [14] }\end{array}$ & $\begin{array}{l}\text { Tg2576 mice, female, } \\
8 \text { months old }\end{array}$ & $\begin{array}{l}\mathrm{EGb} 761(70 \mathrm{mg} / \mathrm{kg} / \text { day }) \\
\text { orally for } 6 \text { months }\end{array}$ & $\begin{array}{l}\text { 1. Morris water maze test } \\
\text { 2. Fibrillar and soluble } \\
\beta \text {-amyloid and protein } \\
\text { oxidation products (ELISA) } \\
\text { 3. Histological assessment } \\
\text { 4. Protein carbonyl }\end{array}$ & $\begin{array}{l}\downarrow \text { average distance to the } \\
\text { platform } \\
\uparrow \text { search ratio } \\
\downarrow \text { soluble } \beta \text {-amyloid } \\
\downarrow \beta \text {-amyloid } \\
\uparrow \text { protein carbonyls }\end{array}$ & $\begin{array}{l}\text { N/A } \\
\text { Antioxidant properties }\end{array}$ \\
\hline $\begin{array}{l}\text { Gong, Q. H. et al. } \\
\quad(2005) \text { [15] }\end{array}$ & $\begin{array}{c}\text { Wistar rats, male, } \\
\text { 8-12 weeks old, daily } \mathrm{AlCl}_{3} \\
\text { solution, } \\
(500 \mathrm{mg} / \mathrm{kg}, \mathrm{i} . \mathrm{g}, 0.5 \mathrm{~mL} / 100 \mathrm{~g}) \\
\text { gavage for } 1 \text { month }\end{array}$ & $\begin{array}{c}\text { EGb761 } \\
(50 \mathrm{mg} / \mathrm{kg} / \text { day, } \\
100 \mathrm{mg} / \mathrm{kg} / \text { day, } \\
200 \mathrm{mg} / \mathrm{kg} / \text { day), } \\
\text { orally for } 2 \text { months }\end{array}$ & $\begin{array}{l}\text { 1. Morris water maze test } \\
\text { 2. Level of caspase-3 } \\
\text { 3. Level of APP } \\
\text { (immunohistochemistry) }\end{array}$ & $\begin{array}{l}\downarrow \text { searching distance } \\
\downarrow \text { escape latency } \\
\downarrow \text { caspase-3 } \\
\downarrow \text { APP }\end{array}$ & $\begin{array}{l}\text { Ameliorated the learning } \\
\text { and memory abilities } \\
\text { Antiapoptosis } \\
\text { N/A }\end{array}$ \\
\hline $\begin{array}{c}\text { Wang, Y. et al. (2006) } \\
\text { [16] }\end{array}$ & $\begin{array}{l}\text { 1. Wistar rats, male, } \\
\text { 12-13 weeks old } \\
\text { 2. Wistar rats, male, } \\
\text { 74-78 weeks old (aged) }\end{array}$ & $\begin{array}{l}\text { 1. EGb761 (30 mg/kg/day) } \\
\text { 2. EGb761 (60 mg/kg/day), } \\
\text { orally for } 30 \text { consecutive days }\end{array}$ & $\begin{array}{l}\text { 1. Morris water maze test } \\
\text { 2. Changes in synaptic } \\
\text { plasticity }\end{array}$ & $\begin{array}{l}\downarrow \text { escape latency } \\
\uparrow \text { search time } \\
\uparrow \text { hippocampal LTP }\end{array}$ & $\begin{array}{l}\text { Improved spatial learning } \\
\text { in aged animals } \\
\text { N/A }\end{array}$ \\
\hline $\begin{array}{l}\text { Gong, Q. H. et al. } \\
\text { (2006) [17] }\end{array}$ & $\begin{array}{l}\text { Wistar rats, male, } 8-12 \text { weeks old, } \\
\text { daily } 50 \mathrm{~g} / \mathrm{L} \mathrm{AICI}_{3} \text {, gavage for } \\
2 \text { months }\end{array}$ & $\begin{array}{c}\text { EGb761 (50 mg/kg/day, } \\
100 \mathrm{mg} / \mathrm{kg} / \text { day, } \\
200 \mathrm{mg} / \mathrm{kg} / \text { day }) \\
\text { orally for } 2 \text { months }\end{array}$ & $\begin{array}{l}\text { 1. Morris water maze test } \\
\text { 2. Effect of AChE activity }\end{array}$ & $\begin{array}{l}\downarrow \text { escape latency } \\
\downarrow \text { searching distance } \\
\downarrow \text { AChE activity }\end{array}$ & $\begin{array}{l}\text { Reduced learning and } \\
\text { memory deficits } \\
\text { Cholinergic improvement }\end{array}$ \\
\hline $\begin{array}{l}\text { Tchantchou, F. et al. } \\
\text { (2007) [18] }\end{array}$ & $\begin{array}{l}\text { 1. TgAPP/PS1 founder mice, } \\
6 \text { months old } \\
\text { 2. TgAPP/PS1 founder mice, } \\
22 \text { months old }\end{array}$ & $\begin{array}{c}\text { EGb761 } \\
\text { (100 mg/kg/day), } \\
\text { orally for } 1 \text { month }\end{array}$ & $\begin{array}{l}\text { 1. Determine the neurogenic } \\
\text { potential } \\
\text { 2. Levels of } A \beta \text { and } \\
\text { CREB/pCREB }\end{array}$ & $\begin{array}{l}\uparrow c e l l \text { proliferation in the } \\
\text { hippocampus } \\
\downarrow A \beta \text { oligomers } \\
\uparrow p C R E B \text { levels in the } \\
\text { hippocampus }\end{array}$ & $\begin{array}{l}\text { Induced neurogenesis as } \\
\text { compensation } \\
\text { N/A }\end{array}$ \\
\hline
\end{tabular}


Table 3. Cont.

\begin{tabular}{|c|c|c|c|c|c|}
\hline Author & Animal Model & Treatment & Method & Result & Mechanism of Action \\
\hline $\begin{array}{l}\text { Blecharz-Klin, K. et al. } \\
\text { (2009) [19] }\end{array}$ & $\begin{array}{l}\text { Wistar rats, male } \\
18 \text { months old }\end{array}$ & $\begin{array}{l}\text { 1. EGb761 (50 mg/kg } \\
\text { b.w./day); } \\
\text { 2. EGb761 (100 mg/kg } \\
\text { b.w./day); } \\
\text { 3. EGb761 (150 mg/kg } \\
\text { b.w./day); } \\
\text { orally for } 3 \text { months }\end{array}$ & $\begin{array}{l}\text { 1. Morris water maze test } \\
\text { 2. Hole-board test } \\
\text { 3. HPLC detects the levels of } \\
\text { DA, 5-HT, NA, and HVA }\end{array}$ & $\begin{array}{l}\downarrow \text { crossings } \\
\downarrow \text { escape latency } \\
\downarrow \text { mean swimming speed } \\
\uparrow \text { motor activity } \\
\uparrow N A \text { in prefrontal cortex and } \\
\text { hippocampus } \\
\downarrow \text { DA in prefrontal cortex and } \\
\text { hippocampus } \\
\uparrow \text { DOPAC in the prefrontal } \\
\text { cortex } \\
\downarrow \text { DOPAC in hippocampus } \\
\uparrow 5 \text {-HT in the striatum }\end{array}$ & Improved spatial memory \\
\hline $\begin{array}{l}\text { Hou, Y. et al. } \\
\text { (2010) [20] }\end{array}$ & $\begin{array}{l}\text { TgAPP/PS1 mice, male, } \\
8 \text { months old }\end{array}$ & $\begin{array}{c}\text { 1. Ginkgo biloba extract } \\
\text { (50 mg/ kg/day),gavage for } \\
4 \text { months; } \\
\text { 2. flavonol ( } 50 \mathrm{mg} / \mathrm{kg} / \text { day), i.p. } \\
\text { for } 7 \text { days }\end{array}$ & $\begin{array}{l}\text { 1. Morris water maze test } \\
\text { 2. Levels of BDNF, pCREB, } \\
\text { and } A \beta \\
\text { 3. Immunohistochemistry of } \\
A \beta \text { deposition }\end{array}$ & $\begin{array}{l}\downarrow \text { time needed to find the } \\
\text { platform } \\
\uparrow \mathrm{BDNF} \text { in neurons and } \\
\text { hippocampus } \\
\downarrow \text { both intracellular and } \\
\text { medium } A \beta \text { levels } \\
\downarrow \mathrm{A} \beta \text { deposition and plaque } \\
\text { formation in hippocampus }\end{array}$ & $\begin{array}{l}\text { Improved impaired spatial } \\
\text { learning } \\
\text { NMDA receptor Antagonist } \\
\text { Anti-inflammatory activity } \\
\text { N/A }\end{array}$ \\
\hline $\begin{array}{l}\text { Tian, X. et al. } \\
\text { (2012) [21] }\end{array}$ & $\begin{array}{l}\text { Sprague-Dawley rats, male, } \\
\text { 3-4 months old, } \\
\text { A } 325-35(1 \mu \mathrm{g} / \mu \mathrm{L}) \text {, i.c.v. }\end{array}$ & $\begin{array}{l}\text { EGb761 (40 mg/tablets) } \\
\text { gavage for } 20 \text { days }\end{array}$ & $\begin{array}{l}\text { 2. Histopathological changes } \\
\text { in } \mathrm{A} \beta \\
\text { 3. Activity of SOD, MDA, } \\
\text { and NO }\end{array}$ & $\begin{array}{l}\downarrow \text { escape latencies } \\
\uparrow \text { platform crossing times } \\
\uparrow \text { percentage of swimming } \\
\text { time in Quadrant } 1 \\
\downarrow \text { density of the damaged } \\
\text { neurons } \\
\uparrow \text { neuronal number } \\
\downarrow \text { SOD } \\
\downarrow \text { MDA } \\
\downarrow N O\end{array}$ & $\begin{array}{l}\text { Improved the learning and } \\
\text { memory cognition } \\
\text { Anti-inflammatory activity }\end{array}$ \\
\hline
\end{tabular}


Table 3. Cont.

\begin{tabular}{|c|c|c|c|c|c|}
\hline Author & Animal Model & Treatment & Method & Result & Mechanism of Action \\
\hline \multirow{7}{*}{$\begin{array}{c}\text { Tian, X. et al. (2013) } \\
\text { [22] }\end{array}$} & \multirow{7}{*}{$\begin{array}{l}\text { Sprague-Dawley rats, male, } \\
4-5 \text { months old, } \\
\text { A } \beta 25-35(1 \mu \mathrm{g} / \mu \mathrm{L}) \text {, i.c.v. }\end{array}$} & \multirow{7}{*}{$\begin{array}{l}\text { EGB761 }(20 \mathrm{mg} / \mathrm{kg} / \text { day }) \\
\text { gavage for } 20 \text { days }\end{array}$} & 1. Morris water maze test & \multirow{7}{*}{$\begin{array}{l}\downarrow \text { escape latency } \\
\uparrow \text { platform crossings } \\
\downarrow \text { SOD } \\
\uparrow \text { GSH } \\
\downarrow \text { MDA } \\
\downarrow \text { caspase- } 9 \\
\downarrow \text { caspase- } 3 \\
\downarrow \text { neuronal apoptosis } \\
\text { and Bax } \\
\uparrow \text { Bcl- } 2 \\
\downarrow \text { Bax }\end{array}$} & \multirow[t]{2}{*}{$\begin{array}{l}\text { Improved the learning and } \\
\text { memory cognition }\end{array}$} \\
\hline & & & 2. Levels of SOD, GSH, and & & \\
\hline & & & MDA & & Antioxidant properties \\
\hline & & & 3. Levels of caspase- 9 and & & \multirow{3}{*}{ Inhibited cell apoptosis } \\
\hline & & & caspase-3 & & \\
\hline & & & 4. TUNEL staining & & \\
\hline & & & 5. RT-PCR of Bcl-2 & & Inhibited cell apoptosis \\
\hline $\begin{array}{l}\text { Jahanshahi, M. et al. } \\
\text { (2013) [23] }\end{array}$ & $\begin{array}{l}\text { Wistar rats, male, Scopolamine } \\
(3 \mathrm{mg} / \mathrm{kg}) \\
\text { intraperitoneal injection }\end{array}$ & $\begin{array}{c}\text { Ginkgo biloba extract } \\
(40 \text { and } 80 \mathrm{mg} / \mathrm{kg}, \mathrm{IP}), \\
\text { everyday injection for a week }\end{array}$ & 1. TUNEL staining & $\begin{array}{l}\downarrow \text { apoptotic cells in the } \\
\text { hippocampus }\end{array}$ & $\begin{array}{l}\text { Antioxidant and hydroxyl } \\
\text { radical scavenging activity }\end{array}$ \\
\hline \multirow[t]{3}{*}{$\begin{array}{l}\text { Zhang, L.-D. et al. } \\
\text { (2015) [24] }\end{array}$} & \multirow[t]{3}{*}{$\begin{array}{l}\text { Sprague-Dawley rats, male, } \\
\text { 5-6 months old, } \\
\text { A } 325-35(10 \mu \mathrm{L} ; 1 \mathrm{~g} / \mathrm{L}) \text {, i.c.v. }\end{array}$} & \multirow[t]{3}{*}{$\begin{array}{l}\text { EGB761 ( } 20 \mathrm{mg} / \mathrm{kg} / \text { day }) \\
\text { gavage for } 20 \text { days }\end{array}$} & 1. Morris water maze test & \multirow{3}{*}{$\begin{array}{l}\uparrow \text { times of crossing the former } \\
\text { platform } \\
\uparrow \text { percentage of time spent in } \\
\text { the quadrant } \\
\downarrow \text { brown precipitate } \\
\text { (apoptosis identification) } \\
\uparrow p-I K K \alpha / \beta \\
\uparrow p-I \kappa B \alpha \\
\uparrow p-N F \kappa B\end{array}$} & $\begin{array}{l}\text { Improved cognitive and } \\
\text { memory capacities }\end{array}$ \\
\hline & & & 2. TUNEL staining & & Inhibited cell apoptosis \\
\hline & & & $\begin{array}{l}\text { 3. Levels of } p-I K K \alpha / \beta, p-I \kappa B \alpha \text {, } \\
\text { and } p-N F \kappa B\end{array}$ & & Anti-inflammatory activity \\
\hline \multirow{3}{*}{$\begin{array}{l}\text { Liu, X. et al. } \\
\text { (2015) [25] }\end{array}$} & \multirow{3}{*}{$\begin{array}{l}\text { TgCRND8 APP-transgenic mice, } \\
\text { female, } 2 \text { months old }\end{array}$} & \multirow{3}{*}{$\begin{array}{c}\text { EGb761 } \\
(600 \mathrm{mg} / \mathrm{kg} / \text { day })(0.6 \%) \\
\text { orally for } 5 \text { months }\end{array}$} & 1. Barnes maze test & $\begin{array}{l}\downarrow \text { time and } \\
\downarrow \text { distance to reach the escape } \\
\text { chamber }\end{array}$ & $\begin{array}{l}\text { Improved cognitive } \\
\text { function }\end{array}$ \\
\hline & & & $\begin{array}{l}\text { 2. Level of } A \beta \text { (ELISA) }(\%) \\
\text { 3. Immunofluorescent staining } \\
\text { of } A \beta\end{array}$ & $\downarrow \mathrm{A} \beta$ & $\mathrm{N} / \mathrm{A}$ \\
\hline & & & $\begin{array}{l}\text { 4. Histological analysis of Iba1 } \\
\text { 5. Levels of tnf- } \alpha, \text { il- } 1 \beta, \text { ccl-2, } \\
\text { and IL-10 }\end{array}$ & $\begin{array}{l}\downarrow \text { Iba1 positive cell number } \\
\downarrow \text { TNF- } \alpha, \text { IL- } 1 \beta, \text { ccl- } 2 \text {, iNOS, } \\
\text { and IL-10 }\end{array}$ & $\begin{array}{l}\text { Neuroinflammatory } \\
\text { inhibition }\end{array}$ \\
\hline
\end{tabular}


Table 3. Cont.

\begin{tabular}{|c|c|c|c|c|c|}
\hline Author & Animal Model & Treatment & Method & Result & Mechanism of Action \\
\hline $\begin{array}{l}\text { Wan, W. et al. } \\
\text { (2016) [26] }\end{array}$ & $\begin{array}{l}\text { APP/PS1 transgenic mice, male, } \\
2 \text { months old }\end{array}$ & $\begin{array}{l}\text { EGb761 (50 mg/kg/day), } \\
\text { orally for } 6 \text { months }\end{array}$ & $\begin{array}{l}\text { 1. Morris water maze test } \\
\text { 2. Level of A } \beta \text { (ELISA) } \\
\text { 3. Ratio of fluorescence } \\
\text { intensity }\end{array}$ & $\begin{array}{l}\downarrow \text { escape latency } \\
\downarrow \text { time of passing the platform } \\
\uparrow \text { crossing times } \\
\downarrow \mathrm{A} \beta \\
\uparrow \text { microglia around the plaque }\end{array}$ & $\begin{array}{l}\text { Improved cognitive } \\
\text { function } \\
\mathrm{N} / \mathrm{A} \\
\text { Attenuated inflammatory } \\
\text { reactions }\end{array}$ \\
\hline $\begin{array}{l}\text { Zeng, K. et al. } \\
\text { (2018) [27] }\end{array}$ & $\begin{array}{c}\text { Sprague-Dawley rats, male, } \\
8 \text { weeks old, Hhcy } \\
\text { (400 } \mu \mathrm{g} / \mathrm{kg} / \text { day }) \text {, for } 14 \text { days i.p. }\end{array}$ & $\begin{array}{l}\text { EGb761 }(400 \mathrm{mg} / \mathrm{kg} / \text { day }) \\
\text { gavage for } 7 \text { days }\end{array}$ & $\begin{array}{l}\text { 1. Morris water maze test } \\
\text { 2. Levels of SOD and MDA } \\
\text { 3. Levels of tau } \\
\text { phosphorylation, PSD95, and } \\
\text { synapsin-1 }\end{array}$ & $\begin{array}{l}\downarrow \text { escape latency } \\
\downarrow \text { SOD } \downarrow \text { MDA } \\
\downarrow \text { tau phosphorylation } \\
\uparrow \text { PSD95 } \\
\uparrow \text { synapsin- } 1\end{array}$ & $\begin{array}{l}\text { Ameliorated memory } \\
\text { deficits } \\
\text { Antioxidant properties } \\
\text { Attenuated oxidative } \\
\text { damage }\end{array}$ \\
\hline $\begin{array}{l}\text { Verma, S. et al. } \\
\text { (2019) [28] }\end{array}$ & $\begin{array}{c}\text { Sprague-Dawley rats, female, } \\
12 \text { months old, } \mathrm{Al}(\mathrm{lac})_{3}(10 \mathrm{mg} / \mathrm{kg} \\
\text { b.wt), daily for } 6 \text { weeks }\end{array}$ & $\begin{array}{c}\text { Ginkgo biloba extract, EGb761 } \\
(100 \mathrm{mg} / \mathrm{kg} / \text { day }) \\
\text { orally for } 6 \text { weeks }\end{array}$ & $\begin{array}{l}\text { 1. Morris water maze test } \\
\text { 2. Histopathological changes } \\
\text { in A } \beta \\
\text { 3. Levels of } 5-\mathrm{HT}, \mathrm{GSH}, \mathrm{GST} \text {, } \\
\text { and SOD } \\
\text { 4. AChE activity }\end{array}$ & $\begin{array}{l}\downarrow \text { time to find the platform } \\
\downarrow \text { escape latency } \\
\downarrow \text { ThT positive cells in } \\
\text { hippocampusand cortex } \\
\downarrow \text { Congo red } \\
\uparrow 5 \text {-HT } \downarrow \text { SOD } \uparrow G S H \downarrow G S T \\
\downarrow \text { AChE activity in the } \\
\text { hippocampus and cortex }\end{array}$ & Improved spatial memory \\
\hline $\begin{array}{l}\text { Verma, S. et al. } \\
\text { (2020) [29] }\end{array}$ & $\begin{array}{c}\text { Sprague-Dawley rats, female, } \\
12 \text { months old, } \mathrm{Al}(\mathrm{lac})_{3} \\
\text { (10 mg/kg b.wt), daily for } 6 \text { weeks }\end{array}$ & $\begin{array}{c}\text { Ginkgo biloba extract, EGb761 } \\
\text { (100 mg/kg/day), } \\
\text { orally for } 6 \text { weeks }\end{array}$ & $\begin{array}{l}\text { 4. Histopathological changes } \\
\text { 5. AchE activity } \\
\text { 6. Level of MAO-B } \\
\text { 7. Immunohistochemistry of } \\
\text { A }(17-23)\end{array}$ & $\begin{array}{l}\downarrow \text { escape latency } \\
\downarrow \text { ROS } \\
\downarrow \text { APP } \\
\downarrow \text { A } \beta \\
\downarrow \text { p-Tau } \\
\downarrow \text { silver positive deposits in } \\
\text { CA1, CA3 } \\
\downarrow \text { congo red positive deposits } \\
\text { in CA1, CA3 } \\
\downarrow \text { ThT positive deposits } \\
\downarrow \text { AChE enzyme activity } \\
\downarrow \text { MAO-B enzyme activity } \\
\downarrow \text { A } \beta \text { (17-23) }\end{array}$ & $\begin{array}{l}\text { Prevented behavioral } \\
\text { impairments } \\
\text { Antioxidative stress } \\
\text { N/A }\end{array}$ \\
\hline
\end{tabular}


Table 4. Characteristics of included clinical studies related to GBE.

\begin{tabular}{|c|c|c|c|c|c|c|c|c|c|c|c|c|}
\hline $\begin{array}{c}\text { Study } \\
\text { Author, Date }\end{array}$ & Country & Inclusion Criteria & Setting & Duration & Treatment & Groups & Age & \multicolumn{4}{|c|}{ Baseline Scale } & $\begin{array}{c}\text { Withdrawal } \\
\text { Rate }\end{array}$ \\
\hline & & & & & & & & \multicolumn{2}{|c|}{ Cognition } & Age & $\underset{(\%)}{\text { Female }}$ & \\
\hline \multicolumn{13}{|c|}{ Effective } \\
\hline $\begin{array}{l}\text { Schaffler and } \\
\text { Reeh, } 1985 \text { [30] }\end{array}$ & $\begin{array}{l}\text { United } \\
\text { Kingdom }\end{array}$ & / & $\begin{array}{c}\text { Normal } \\
\text { healthy } \\
\text { volunteers }\end{array}$ & $\begin{array}{c}2 \\
\text { weeks }\end{array}$ & $\begin{array}{l}\text { EGB (Tebonin) } \\
80 \text { mg/day }\end{array}$ & $\begin{array}{c}\text { EGB: } n=4 \\
\text { Placebo: } n=4\end{array}$ & 27 & / & / & $27.3 \pm 2.6$ & 0 & / \\
\hline $\begin{array}{l}\text { Wesnes et al., } \\
1987 \text { [31] }\end{array}$ & $\begin{array}{l}\text { United } \\
\text { Kingdom }\end{array}$ & $\begin{array}{c}\text { Crichton geriatric } \\
\text { behavioral scale }>14\end{array}$ & Outpatient & $\begin{array}{c}12 \\
\text { weeks }\end{array}$ & $\begin{array}{l}\text { EGB (Tanakan) } \\
120 \text { mg/day }\end{array}$ & $\begin{array}{c}\text { EGB: } n=27 \\
\text { Placebo: } n=27\end{array}$ & $62 \sim 85$ & / & / & $\begin{array}{l}70.7 \pm 7.1 \\
71.3 \pm 6.6\end{array}$ & $\begin{array}{l}30 \% \\
44 \%\end{array}$ & $7 \%$ \\
\hline $\begin{array}{c}\text { Kanowski, } \\
\text { Nerrmann, } \\
\text { et al., } 1996 \text { [33] }\end{array}$ & Germany & $\begin{array}{c}\text { SKT: } 6 \sim 18 ; \\
\text { MMSE: } 13 \sim 25\end{array}$ & Outpatient & $\begin{array}{c}24 \\
\text { weeks }\end{array}$ & $\begin{array}{c}\text { EGb761 } \\
240 \mathrm{mg} / \text { day }\end{array}$ & $\begin{array}{l}\text { EGb761: } n=79 \\
\text { Placebo: } n=77\end{array}$ & $>55$ & SKT & $\begin{array}{l}10.2 \pm 3.0 \\
11.2 \pm 3.4\end{array}$ & $\begin{array}{l}70 \pm 10 \\
68 \pm 10\end{array}$ & $\begin{array}{l}66 \% \\
69 \%\end{array}$ & $30 \%$ \\
\hline $\begin{array}{l}\text { Maurer, Dierks, } \\
\text { et al., } 1997 \text { [34] }\end{array}$ & Germany & $\begin{array}{c}\text { DSM-III-R and ICD-10 } \\
\text { criteria; Hachinski ischemic } \\
\text { score < } 4 \text { mean; BCRS } \\
\text { score } 3-5\end{array}$ & Outpatient & $\begin{array}{c}12 \\
\text { weeks }\end{array}$ & $\begin{array}{c}\text { EGb761 } \\
240 \mathrm{mg} / \text { day }\end{array}$ & $\begin{array}{l}\text { EGb761: } n=10 \\
\text { Placebo: } n=10\end{array}$ & $50 \sim 80$ & $\begin{array}{c}\text { SKT } \\
\text { ADAS-Cog }\end{array}$ & $\begin{array}{c}19.7 \pm 6.4 \\
18.1 \pm 9.4 \\
31.2 \pm 12.6 \\
36.1 \pm 15.2 \\
\end{array}$ & $\begin{array}{c}68.5 \pm 6 \\
60.6 \pm 8.2\end{array}$ & $\begin{array}{l}56 \% \\
45 \%\end{array}$ & $10 \%$ \\
\hline $\begin{array}{l}\text { Barsa, Kieserc, } \\
\text { et al., } 2000 \text { [35] }\end{array}$ & United States & $\begin{array}{l}\text { DSM-III-R and ICD-10 } \\
\text { criteria; MMSE: 9 26; } \\
\text { global deterioration } \\
\text { scale: } 3 \sim 6\end{array}$ & Outpatient & $\begin{array}{c}26 \\
\text { weeks }\end{array}$ & $\begin{array}{c}\text { EGb761 } \\
120 \mathrm{mg} / \text { day }\end{array}$ & $\begin{array}{l}\text { EGb761: } n=166 \\
\text { Placebo: } n=161\end{array}$ & $>45$ & $\begin{array}{c}\text { MMSE } \\
\text { ADAS-Cog }\end{array}$ & $\begin{array}{c}21.1 \pm 5.8 \\
21.2 \pm 5.5 \\
20.0 \pm 16.0 \\
20.5 \pm 14.7\end{array}$ & $\begin{array}{l}69 \pm 10 \\
69 \pm 10\end{array}$ & $\begin{array}{l}51 \% \\
56 \%\end{array}$ & $21 \%$ \\
\hline $\begin{array}{c}\text { Kanowski and } \\
\text { Hoerr, 2003 } \\
{[36]}\end{array}$ & Germany & $\begin{array}{l}\text { DSM-III-R and ICD-10 } \\
\text { criteria; SKT: } 6 \sim 18 ; \\
\text { MMSE: } 13 \sim 25\end{array}$ & Outpatient & $\begin{array}{c}24 \\
\text { weeks }\end{array}$ & $\begin{array}{c}\text { EGb761 } \\
240 \mathrm{mg} / \text { day }\end{array}$ & $\begin{array}{l}\text { EGb761: } n=106 \\
\text { Placebo: } n=99\end{array}$ & $>55$ & $\begin{array}{c}\text { MMSE } \\
\text { SKT } \\
\text { ADAS-Cog }\end{array}$ & $\begin{array}{l}21.6 \pm 2.6 \\
21.5 \pm 2.4 \\
10.5 \pm 3.2 \\
11.2 \pm 3.3 \\
19.0 \pm 4.1 \\
19.9 \pm 4.3\end{array}$ & $\begin{array}{l}72 \pm 10 \\
72 \pm 10\end{array}$ & $\begin{array}{l}68 \% \\
71 \%\end{array}$ & $7.65 \%$ \\
\hline $\begin{array}{c}\text { Mazza, } \\
\text { Capuano, et al., } \\
2006 \text { [37] }\end{array}$ & Italy & $\begin{array}{l}\text { Brief cognitive } \\
\text { rating scale: } 3 \sim 5 \text {; Hachinski } \\
\text { ischemic score <4; SKT: } \\
\text { 8 23; MMSE: 13 25 }\end{array}$ & Outpatient & $\begin{array}{c}24 \\
\text { weeks }\end{array}$ & $\begin{array}{c}\text { EGb761 } \\
160 \mathrm{mg} / \text { day }\end{array}$ & $\begin{array}{c}\text { EGb761: } n=25 \\
\text { donepezil: } n=25 \\
\text { Placebo: } n=16\end{array}$ & $50 \sim 80$ & $\begin{array}{l}\text { MMSE } \\
\text { SKT }\end{array}$ & $\begin{array}{l}18.8 \pm 3.6 \\
18.8 \pm 3.6 \\
16.5 \pm 3.1 \\
15.9 \pm 3.9\end{array}$ & $\begin{array}{l}66.2 \pm 6 \\
64.5 \pm 6 \\
69.8 \pm 3\end{array}$ & $\begin{array}{l}52 \% \\
48 \% \\
61 \%\end{array}$ & $19.70 \%$ \\
\hline
\end{tabular}


Table 4. Cont.

\begin{tabular}{|c|c|c|c|c|c|c|c|c|c|c|c|c|}
\hline $\begin{array}{c}\text { Study } \\
\text { Author, Date }\end{array}$ & Country & Inclusion Criteria & Setting & Duration & Treatment & Groups & Age & \multicolumn{4}{|c|}{ Baseline Scale } & \multirow[t]{2}{*}{$\begin{array}{c}\text { Withdrawal } \\
\text { Rate }\end{array}$} \\
\hline & & & & & & & & \multicolumn{2}{|c|}{ Cognition } & Age & $\underset{(\%)}{\text { Female }}$ & \\
\hline $\begin{array}{l}\text { Napryeyenko } \\
\text { and Borzenko, } \\
2007 \text { [38] }\end{array}$ & Ukraine & $\begin{array}{l}\text { NINCDS/ADRDA } \\
\text { diagnostic criteria: } \\
\text { SKT: 9 23; MMSE: 14 25; } \\
\text { ADAS-Cog: 17 35 }\end{array}$ & Outpatient & $\begin{array}{c}22 \\
\text { weeks }\end{array}$ & $\begin{array}{c}\text { EGb761 } \\
240 \mathrm{mg} / \text { day }\end{array}$ & $\begin{array}{l}\text { EGb761: } n=198 \\
\text { Placebo: } n=197\end{array}$ & $>50$ & SKT & $\begin{array}{l}15.6 \pm 3.9 \\
15.4 \pm 3.7\end{array}$ & $\begin{array}{l}65 \pm 8 \\
63 \pm 8\end{array}$ & $\begin{array}{l}72 \% \\
72 \%\end{array}$ & $1.25 \%$ \\
\hline $\begin{array}{c}\text { Ihl, } \\
\text { Bachinskaya, } \\
\text { et al., } 2011 \text { [39] }\end{array}$ & Ukraine & $\begin{array}{c}\text { NINCDS-ADRDA } \\
\text { criteria; SKT: 9 23; MMSE: } \\
\text { 14 25; ADAS-Cog: 17 35 }\end{array}$ & Outpatient & $\begin{array}{c}24 \\
\text { weeks }\end{array}$ & $\begin{array}{c}\text { EGb761 } \\
240 \mathrm{mg} / \text { day }\end{array}$ & $\begin{array}{l}\text { EGb761: } n=206 \\
\text { Placebo: } n=204\end{array}$ & $>50$ & SKT & $\begin{array}{l}16.7 \pm 3.9 \\
17.2 \pm 3.7\end{array}$ & $\begin{array}{c}65 \pm 10 \\
65 \pm 9 \\
\end{array}$ & $\begin{array}{l}69 \% \\
66 \%\end{array}$ & $6.82 \%$ \\
\hline $\begin{array}{l}\text { Amieva, } \\
\text { Meillon, et al., } \\
2013 \text { [40] }\end{array}$ & France & / & Outpatient & $\begin{array}{c}20 \\
\text { years }\end{array}$ & $\begin{array}{l}\text { EGb761 dosage } \\
\text { unclear }\end{array}$ & $\begin{array}{c}\text { EGb761: } n=589 \\
\text { Piracetam: } \\
n=149 \\
\text { Placebo: } n=2874 \\
\end{array}$ & $>65$ & MMSE & $\begin{array}{l}26.3 \pm 2.9 \\
25.7 \pm 3.9 \\
25.7 \pm 3.5 \\
\end{array}$ & $\begin{array}{c}74.8 \pm 6.6 \\
75.7 \pm 6.6 \\
75 \pm 6.9 \\
\end{array}$ & $\begin{array}{l}73.9 \% \\
61.1 \% \\
54.1 \%\end{array}$ & 0 \\
\hline $\begin{array}{l}\text { Canevelli, } \\
\text { Adali, et al., } \\
2014 \text { [41] }\end{array}$ & Europe & $\begin{array}{l}\text { NINCDS-ADRDA } \\
\text { criteria, MMSE: } 10 \sim 26\end{array}$ & Outpatients & 1 year & $\begin{array}{c}\text { EGb761 } \\
120 \mathrm{mg} / \text { day }\end{array}$ & $\begin{array}{c}\text { EGb761 + ChEIs: } \\
n=29 \\
\text { ChEIs: } n=799\end{array}$ & $68 \sim 84$ & $\begin{array}{c}\text { MMSE } \\
\text { ADAS-Cog }\end{array}$ & $\begin{array}{l}21.2 \pm 3.5 \\
20.5 \pm 3.9 \\
15.8 \pm 7.9 \\
20.6 \pm 8.9 \\
\end{array}$ & $\begin{array}{c}76.2 \pm 6.87 \\
5.8 \pm 7.8\end{array}$ & $\begin{array}{l}62.1 \% \\
64.8 \%\end{array}$ & 0 \\
\hline $\begin{array}{c}\text { Hoerr and } \\
\text { Nacu, 2016 [42] }\end{array}$ & $\begin{array}{l}\text { Russian } \\
\text { Federation, } \\
\text { Republic of } \\
\text { Belarus, } \\
\text { Republic of } \\
\text { Moldova } \\
\end{array}$ & $\begin{array}{l}\text { SKT: 9 23, mild to } \\
\text { moderate dementia; test for } \\
\text { the early detection of } \\
\text { dementia with } \\
\text { differentiation from } \\
\text { depression } \leq 35\end{array}$ & Outpatient & $\begin{array}{c}24 \\
\text { weeks }\end{array}$ & $\begin{array}{c}\text { EGb761 } \\
240 \mathrm{mg} / \text { day }\end{array}$ & $\begin{array}{l}\text { EGb761: } n=200 \\
\text { Placebo: } n=202\end{array}$ & $>65$ & SKT & $\begin{array}{l}15.1 \pm 4.1 \\
15.3 \pm 4.2\end{array}$ & $\begin{array}{l}65.1 \pm 8.8 \\
64.9 \pm 9.4\end{array}$ & $\begin{array}{l}69.5 \% \\
69.3 \%\end{array}$ & $2 \%$ \\
\hline \multicolumn{13}{|c|}{ Ineffective } \\
\hline $\begin{array}{l}\text { Subhan and } \\
\text { Hindmarch, } \\
1984 \text { [43] }\end{array}$ & $\begin{array}{l}\text { United } \\
\text { Kingdom }\end{array}$ & / & $\begin{array}{l}\text { Normal } \\
\text { healthy } \\
\text { volunteers }\end{array}$ & $1 \mathrm{~h}$ & $\begin{array}{l}\text { EGb } 761 \\
120 \mathrm{mg} / 240 \mathrm{mg} / \\
600 \mathrm{mg}\end{array}$ & $\begin{array}{c}\text { EGb761(120): } \\
n=2 \\
\text { EGb761(240): } \\
n=2 \\
\text { EGb761(600): } \\
n=2 \\
\text { Placebo: } n=2 \\
\end{array}$ & 32 & / & / & $32 \pm 0$ & $100 \%$ & / \\
\hline $\begin{array}{c}\text { Schneider, } \\
\text { DeKosky, et al., } \\
2005 \text { [44] }\end{array}$ & United States & $\begin{array}{c}\text { NINCDS/ } \\
\text { ADRDA criteria; modified } \\
\text { Hachinski ischemic score } \\
\text { <4; MMSE: } 10 \sim 24\end{array}$ & Outpatients & $\begin{array}{c}26 \\
\text { weeks }\end{array}$ & $\begin{array}{l}\text { EGb761 } \\
120 / 240 \mathrm{mg} / \text { day }\end{array}$ & $\begin{array}{c}\text { EGb761(120): } \\
n=169 \\
\text { EGb761(240): } \\
n=170 \\
\text { Placebo: } n=174\end{array}$ & $>60$ & $\begin{array}{c}\text { MMSE } \\
\text { ADAS-Cog }\end{array}$ & $\begin{array}{c}17.4 \pm 3.8(240) \\
17.9 \pm 4.5(120) \\
17.6 \pm 3.9 \\
24.8 \pm 11.3(240) \\
26.8 \pm 13.7(120) \\
26.2 \pm 11.8\end{array}$ & $\begin{array}{l}78.6 \pm 7.0 \\
78.1 \pm 7.0 \\
77.5 \pm 7.4\end{array}$ & $\begin{array}{l}50 \% \\
56 \% \\
52 \%\end{array}$ & $20.00 \%$ \\
\hline
\end{tabular}


Table 4. Cont.

\begin{tabular}{|c|c|c|c|c|c|c|c|c|c|c|c|c|}
\hline \multirow{2}{*}{$\begin{array}{c}\text { Study } \\
\text { Author, Date } \\
\end{array}$} & \multirow[t]{2}{*}{ Country } & \multirow[t]{2}{*}{ Inclusion Criteria } & \multirow[t]{2}{*}{ Setting } & \multirow[t]{2}{*}{ Duration } & \multirow[t]{2}{*}{ Treatment } & \multirow[t]{2}{*}{ Groups } & \multirow[t]{2}{*}{ Age } & \multicolumn{4}{|c|}{ Baseline Scale } & \multirow[t]{2}{*}{$\begin{array}{l}\text { Withdrawal } \\
\text { Rate }\end{array}$} \\
\hline & & & & & & & & \multicolumn{2}{|c|}{ Cognition } & Age & $\underset{(\%)}{\text { Female }}$ & \\
\hline $\begin{array}{l}\text { McCarney, } \\
\text { Fisher, et al., } \\
2008 \text { [45] }\end{array}$ & $\begin{array}{l}\text { United } \\
\text { Kingdom }\end{array}$ & $\begin{array}{l}\text { DSM-IV criteria; MMSE: } \\
\text { 12 26 }\end{array}$ & Outpatient & $\begin{array}{c}24 \\
\text { weeks }\end{array}$ & $\begin{array}{l}\text { EGb761 } 120 \\
\text { mg/day }\end{array}$ & $\begin{array}{l}\text { EGb761: } n=88 \\
\text { Placebo: } n=88\end{array}$ & $>55$ & $\begin{array}{c}\text { MMSE } \\
\text { ADAS-Cog }\end{array}$ & $\begin{array}{c}23 \\
22 \\
20.4 \pm 8.2 \\
25 \pm 10.3 \\
\end{array}$ & $\begin{array}{l}79.3 \pm 7.7 \\
79.7 \pm 7.5 \\
\end{array}$ & $\begin{array}{l}58.0 \% \\
63.6 \%\end{array}$ & $25.60 \%$ \\
\hline $\begin{array}{l}\text { Snitz, O'Meara, } \\
\text { et al., } 2009 \text { [46] }\end{array}$ & United States & $\begin{array}{c}\text { MMSE; } \\
\text { ADAS-Cog; } \\
\text { neuropsychological test }\end{array}$ & $\begin{array}{l}\text { community- } \\
\text { dwelling } \\
\text { partici- } \\
\text { pants }\end{array}$ & $\begin{array}{c}6.1 \\
\text { years }\end{array}$ & $\begin{array}{l}\text { EGb761 } 240 \\
\text { mg/day }\end{array}$ & $\begin{array}{l}\text { EGb761: } n=1545 \\
\text { Placebo: } n=1524\end{array}$ & 72 96 & $\begin{array}{c}\text { MMSE } \\
\text { ADAS-Cog }\end{array}$ & $\begin{array}{c}93.4 \pm 4.7 \\
93.3 \pm 4.7 \\
6.5 \pm 2.86 .4 \pm 2.7\end{array}$ & $\begin{array}{l}79.1 \pm 3.3 \\
79.1 \pm 3.3 \\
\end{array}$ & $\begin{array}{l}45 \% \\
47 \%\end{array}$ & $37.80 \%$ \\
\hline $\begin{array}{l}\text { Vellas, Coley, } \\
\text { et al., } 2012 \text { [47] }\end{array}$ & France & $\begin{array}{c}\text { MMSE: }>25 ; \\
\text { covianxiety scale }<6 ; \\
\text { geriatric depression scale } \\
<15\end{array}$ & Outpatient & 5 years & $\begin{array}{l}\text { EGb761 } 240 \\
\text { mg/day }\end{array}$ & $\begin{array}{l}\text { EGb761: } n=1419 \\
\text { Placebo: } n=1435\end{array}$ & $>70$ & MMSE & $\begin{array}{l}27.6 \pm 1.9 \\
27.6 \pm 1.9\end{array}$ & $\begin{array}{l}76.3 \pm 4.4 \\
76.3 \pm 4.4 \\
\end{array}$ & $\begin{array}{l}67 \% \\
66 \%\end{array}$ & $31 \%$ \\
\hline $\begin{array}{c}\text { Nasab, } \\
\text { Bahrammi, } \\
\text { et al., } 2012 \text { [48] }\end{array}$ & Iran & $\begin{array}{c}\text { DSM IV criteria; } \\
\text { NINCDS-ADRDA criteria; } \\
\text { MMSE: } 10 \sim 24\end{array}$ & Outpatients & $\begin{array}{c}24 \\
\text { weeks }\end{array}$ & $\begin{array}{l}\text { EGb761 } 120 \\
\text { mg/day }\end{array}$ & $\begin{array}{c}\text { EGb761: } n=25 \\
\text { Rivastigmine: } \\
n=25\end{array}$ & $\begin{array}{l}50- \\
75\end{array}$ & MMSE & $\begin{array}{l}15.6 \pm 4.1 \\
16.6 \pm 4.0\end{array}$ & $\begin{array}{l}65.7 \pm 4.7 \\
66.0 \pm 4.6\end{array}$ & $\begin{array}{r}52 \% \\
57.7 \%\end{array}$ & $9.00 \%$ \\
\hline
\end{tabular}




\section{Results}

\subsection{Study Selection}

\subsubsection{Screening of Preclinical Studies}

The basic search produced a total of 1038 articles from the electronic databases, Web of Science, PubMed, and Google Scholar. After browsing the titles and abstracts of the articles, we excluded 200 articles according to the following criteria: (1) Review articles, comments, letters, case reports $(n=305)$; (2) Clinical trials $(n=273)$; and (3) The efficiency of the GBE was tested on AD cell models $(n=43)$. Next, by carefully screening the full texts of the remaining 292 articles, 275 articles were excluded for the following four reasons: (1) The GBE was tested on nonmammalian animal models (zebra fish, Caenorhabditis elegans, drosophila, etc.); (2) The GBE was not tested on an AD model; (3) Other drugs were used in combination with GBE; and (4) The full text was missing. Finally, we included 17 articles. The details are shown in Figure 1.

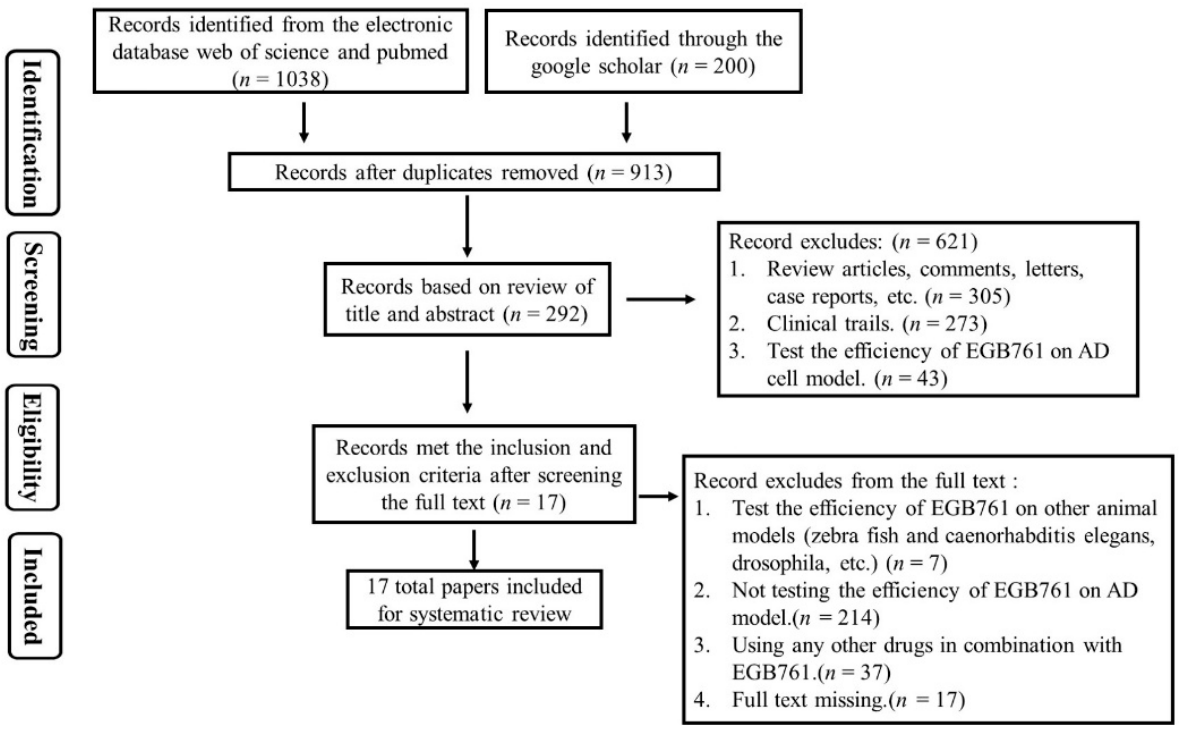

Figure 1. The screening flow chart of the preclinical studies.

\subsubsection{Recruitment Status of GBE Clinical Trials}

As is shown in the Figure 2, a total of 98 clinical trials related to G. biloba were identified in the ClinicalTrials.gov website or in Google Scholar. More than half of those clinical trials have been completed $(n=57)$. The statuses of the remainder were classified as: unknown $(n=13)$; active, not recruiting $(n=2)$; terminated $(n=3)$; withdrawn $(n=3)$; enrolling by invitation $(n=1)$; recruiting $(n=14)$; and not yet recruiting $(n=5)$. In this systematic review, 20 clinical-trial-related articles have been included in order to conduct a further meta-analysis and a methodological quality assessment.

\subsection{Article Characteristics}

\subsubsection{Analysis of Included Preclinical Studies}

In the preclinical studies reported in the 17 articles, two animal species were used: mice $(n=6)$, and rats $(n=11)$. Four strains of mice were used: C57BL/6 mice $(n=1)$ [12], Tg2576 mice $(n=1)$ [14], TgAPP/PS1 mice $(n=3)[18,26,50]$, and TgCRND8 APP-transgenic mice $(n=1)$ [25]. Two strains of rats were used: Wistar rats $(n=5)[15-17,19,23]$, and Sprague-Dawley rats $(n=6)[21,22,24,27-29]$. In twelve studies, only male animals were used, while, in four studies, only female animals were used; one article did not mention the sex of the animals. The animal numbers (from 8 to 36) and the animal ages varied greatly among the articles. The details are shown in Table 3.

Half of the studies established their AD models by using drugs, such as $\mathrm{AlCl}_{3}$ $(n=4)[15,17,28,29]$, scopolamine $(n=1)$ [23], hyperhomocysteinemia $(n=1)$ [27], and 
$\mathrm{A} \beta^{25-35}(n=3)[21,22,24]$. These toxin-induced AD models have a common feature of mimicking the pathological alterations and cognitive impairment of $\mathrm{AD}$, but each has its drawbacks. Specifically, $\mathrm{AlCl}_{3}$ (aluminum) has a significant effect on the enzyme activity, which influences protein synthesis and the neurotransmitter activity, and moreover, this method spends a long time on modeling. Scopolamine is a well-known M-cholinergic receptor blocker, which can damage the cholinergic neuron. However, scopolamine-treated animals lack the typical pathological features of $\mathrm{AD}$; that is, scopolamine fails to induce the irreversible nerve damage in $\mathrm{AD}$. $\mathrm{A} \beta$ injection causes an accumulation of $\mathrm{A} \beta$, leading to plaque formation and neuron toxicity. However, this acute toxicity model cannot reflect the relatively slow neurodegeneration process of AD. Hyperhomocysteinemia seems applicable for AD modeling, and can significantly increase the plasma Hcy levels for memory impairment and tau hyperphosphorylation in rats.

Transgenic mice were used as AD models in five studies (29.4\%), and wild-type naturally aging animals were used in the remaining three studies (17.6\%). Transgenic mice, including Tg2576 mice, TgAPP/PS1 mice, and TgCRND8 APP-transgenic mice, are suitable to represent $\mathrm{AD}$ pathogenesis, and have been widely used for pharmacological testing in preclinical studies. In some studies, researchers chose the natural aging model, with the characteristics of late onset in actual AD patients. This model can better reflect the therapeutic effects of drugs, but it fails to simulate the key pathological manifestations of $\mathrm{AD}$, including amyloid plaques and NFT.

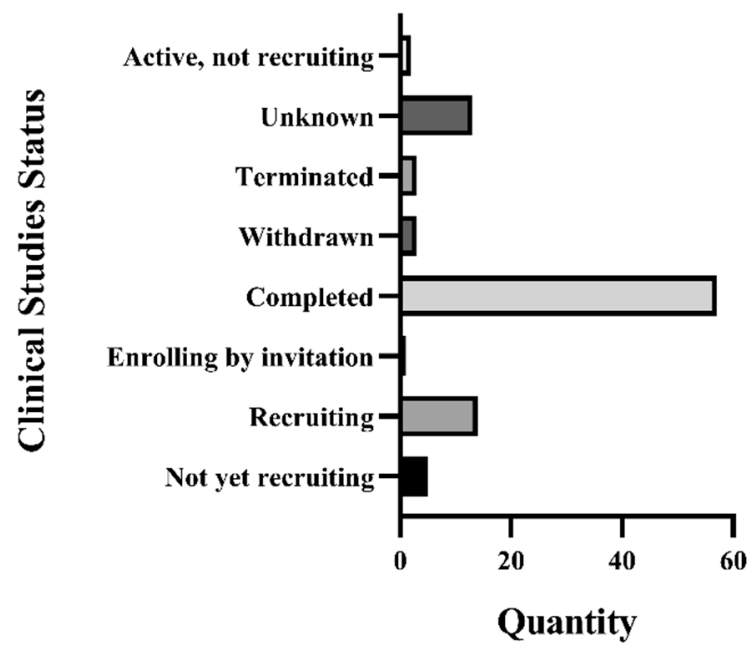

Figure 2. Recruitment status of GBE clinical trials.

\subsubsection{Analysis of Included Clinical Studies}

A total of 14 of the 20 included clinical studies (70.0\%) conclude that GBE can effectively improve the cognitive ability of AD patients, while others report no significant improvement. The criteria used in the 20 clinical studies were: DSM-III-R criteria [51]; ICD-10 criteria [52]; the Mini-Mental State Examination (MMSE) [53]; the Alzheimer's Disease Assessment Scale Cognitive Subscale (ADAS-Cog) [54]; the Global Deterioration Scale score, NINCDS-ADRDA criteria [55]; and the Syndom Kurztest (SKT) [20]. The baseline scales of each clinical trial are listed in Table 4, including the cognition conditions (SKT/MMSE/ADAS-Cog), ages, and sex ratios. Among the 14 effective studies, $7(50.0 \%)$ chose a GBE dose of $240 \mathrm{mg}$ per day [8,33,34,36,38,39,42]; $4(28.6 \%)$ chose a dose of $120 \mathrm{mg} /$ day [31,32,35,41]; 1 selected $160 \mathrm{mg} /$ day [37], and 1 selected $80 \mathrm{mg} /$ day [30]. The dose used in the remaining study was unclear [40]. In the included effective studies, the duration of the drug administration ranged from 2 weeks to 20 years. Interestingly, 10 studies $(71.4 \%)$ chose a relatively long drug administration period (over 20 weeks) [8,32,33,35-39,41,42]. A total of two clinical studies tracked 12 weeks [31,34], one clinical study lasted for 20 years [40], and the other one lasted for only 2 weeks [30]. According to the raw data from the clinical trials, the comparison of the GBE effects on 
$\mathrm{AD}$ symptoms was made according to the age groups (Figure. $3 \mathrm{~A}$ ). The age groups of $\mathrm{AD}$ patients that showed effective outcomes were mainly distributed in the 60-70-year-old ( $n=544)$ and $70-80$-year-old $(n=588)$ groups. Most of the AD patients who showed no significant improvement for years after GBE treatment were over the age of 70 (70-80: $n=2116$; 80-90: $n=1072$ ) (Figure 3A). Actually, when we calculate the effective ratio among the different age groups, we find an obvious decline with increasing age, indicating that GBE may be more effective in younger populations, whose AD-related damage is normally mild. The details are shown in Figure 3B.

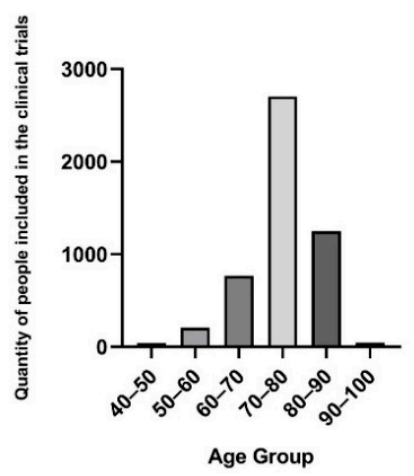

(A)

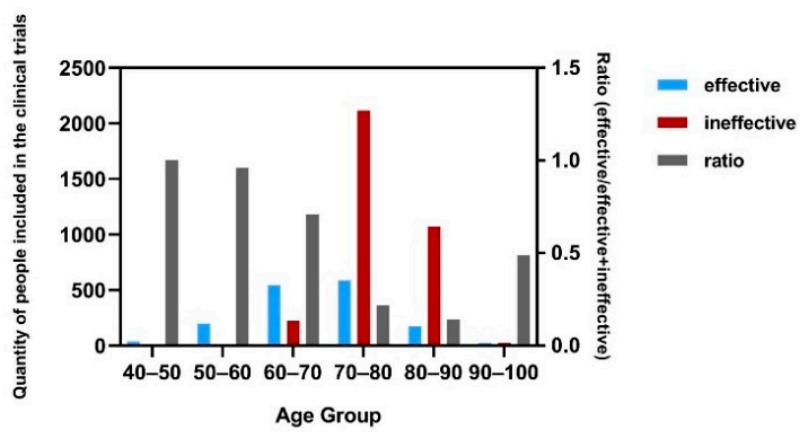

(B)

Figure 3. (A) The age distribution of all participants in clinical trials. (B) Comparison of age groups in clinical trial studies.

In the studies that reported negative outcomes, only two of six studies were conducted over periods of five years [46,47], and the majority of the patients in these two studies were over 75 years old. We speculate that, in the elderly population, the disease has progressed to an irreversible condition that cannot be alleviated by GBE administration. Another failed clinical trial lasted for only six weeks [56]. Schneider et al. (2005) [44] conducted a randomized placebo-controlled double-blind trial with GBE administration for 26 weeks. However, when they found a lack of cognitive decline in patients taking a placebo, they suspected that the assessment criteria may not have been sensitive enough to detect a treatment effect, and they considered the results inconclusive. Similarly, McCarney et al. (2008) [45] and Nasab et al. (2012) [48] conducted trials with daily administrations of $120 \mathrm{mg}$ GBE for 24 weeks. Nasab compared the efficacy of GBE with that of rivastigmine. Both trials confirmed the safety of GBE, but Nasab considered that rivastigmine, a representative cholinesterase inhibitor, performed better than GBE in AD treatment. Nonetheless, the result of the clinical trial was considered insignificant. All three trials had subpositive results with ambiguous conclusions. The details are shown in Table 4.

\subsection{Meta-Analysis Results}

\subsubsection{Behavioral Test Analysis in Preclinical Studies}

The Morris water maze (MWM) test is a classical experiment used to evaluate the cognitive functions of learning and memory in AD animal models [57]. The time of crossing to the platform indicates the spatial memory ability, in terms of the memory storage and extraction capability, while the escape latency in the spatial probe test is considered to be an indicator of the spatial learning ability.

A total of 4 of the $17(23.5 \%)$ articles detected the numbers of times crossing the target quadrant in the MWM to evaluate the spatial memory in the experimental AD animals. A total of 42 animals (rats and mice, $n=32$ and $n=10$, respectively) were included and treated with different doses of GBE $(100 \mathrm{mg} / \mathrm{kg}$ in one article, $50 \mathrm{mg} / \mathrm{kg}$ in one article, $40 \mathrm{mg} / \mathrm{kg}$ in one article, and $20 \mathrm{mg} / \mathrm{kg}$ in one article). A total of 42 animals (rats and mice, $n=32$ and $n=10$, respectively) were treated as control groups. The univariate statistical analysis was conducted using Revman 5.3 software. In the experimental AD model, whether it 
was mice or rats, GBE significantly increased the numbers of times animals crossed the target quadrant, when compared with vehicle-treated animals $(p<0.00001)$, with a Std. mean difference of 2.79. Notably, the results of the forest plot show moderate heterogeneity $\left(p=0.02, \mathrm{I}^{2}=69 \%\right)$, which indicates that GBE has a potential relationship with the increased crossing times of the AD animal model. The details are shown in Figure 4.

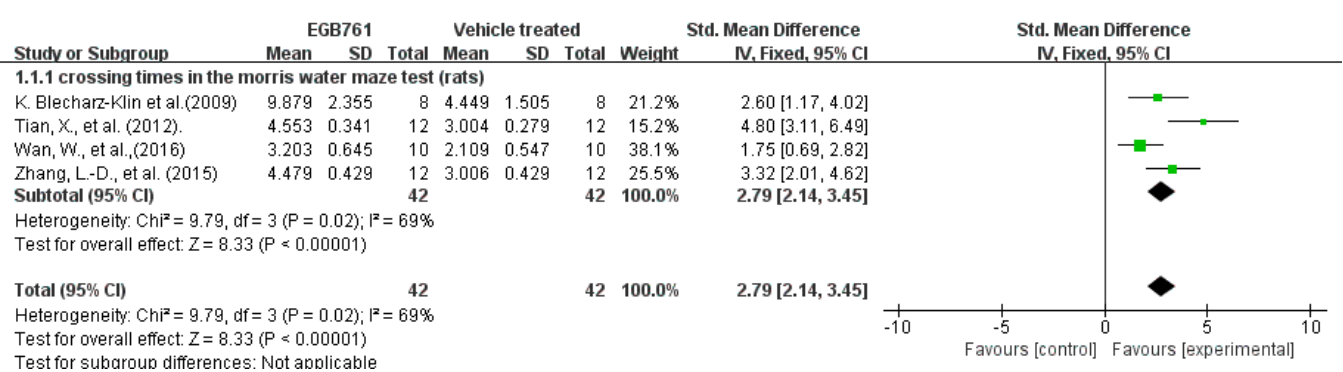

Figure 4. Forest plot for comparison: GBE treatment versus vehicle treatment. Outcome: numbers of times crossing the target quadrant in MWM. The square area represents the weight assigned to the study in the meta-analysis. The horizontal line represents the $95 \%$ confidence interval.

A total of 6 of the 17 (35\%) articles tested the escape latency in the spatial probe test of the MWM to assess the spatial learning ability in the experimental AD animals. A total of 45 animals (rats, $n=31$, mice, $n=14$, respectively) were included and treated with different doses of GBE (100 mg/ kg in two articles, $400 \mathrm{mg} / \mathrm{kg}$ in one article, $60 \mathrm{mg} / \mathrm{kg}$ in one article, and $50 \mathrm{mg} / \mathrm{kg}$ in two articles). The univariate statistical analysis was conducted using Revman 5.3 software. In a forest plot, GBE showed a strong curative effect in decreasing the escape latency $(p<0.00001)$, compared with vehicle treatment in the MWM, for both mice and rats, with a mean difference (MD) between 30.54 and 40.02. Moreover, the subgroup outcomes of the forest plot show no heterogeneity $\left(\mathrm{I}^{2}=0 \%, p=0.56, \mathrm{I}^{2}=0, p=0.92\right)$. The results of the combination analysis indicate that GBE improved behavior and cognitive impairment $(p<0.00001)$, with moderate heterogeneity $\left(\mathrm{I}^{2}=74 \%, p=0.001\right)$. Differences in the drug dosages, the methods of measurement, and the strains of animals are possible reasons for the higher heterogeneity. The details are shown in Figure 5.

\subsubsection{Cognition Improvement Analysis in Clinical Studies}

The Syndom Kurztest (SKT) and the Alzheimer's Disease Assessment Scale Cognitive Subscale (ADAS-Cog) are two widely used cognitive tests that can accurately reflect the attention and memory deficits of AD patients $[20,54]$. The cognition tests, such as SKT and ADAS-Cog, were evaluated at the beginnings and the ends of the clinical trials. A total of 7 of $10(70 \%)$ effective studies were included in the meta-analysis of AD patients in the SKT. A total of 819 patients who had been diagnosed with AD, or that had AD-like symptoms, received lower SKT scores after GBE treatment for 12 to 24 weeks. The univariate statistical analysis was conducted using Revman 5.3 software. In the forest plot, the changes in the SKT scores ranged from -3.2 to -0.0075 in the GBE treatment group, compared with -1.2 to 1.3 in the placebo group, with SMD $(95 \% \mathrm{CI})=-2.44[-2.60,-2.29]$. Although the study results show that GBE can ameliorate cognitive deficits, the forest plot shows that high heterogeneity $\left(p<0.00001, \mathrm{I}^{2}=99 \%\right)$, different drug dosages, and different administration times are possible reasons for the differences between the experimental and placebo groups. The details are shown in Figure 6.

Next, the subgroup analysis of the SKT by dose included six effective studies. A total of 779 patients diagnosed with AD or cognitive impairment were treated with GBE (daily dose below $240 \mathrm{mg}, n=104$; daily dose of $240 \mathrm{mg}, n=675$ ), while 703 patients were treated with placebos (daily dose below $240 \mathrm{mg}, n=70$; daily dose of $240 \mathrm{mg}, n=633$ ). The univariate statistical analysis was conducted using Revman 5.3 software. In the forest plot, the changes in the SKT scores ranged from -3.3 to -0.7 in the GBE groups at doses below $240 \mathrm{mg} / \mathrm{day}$, and from -1.2 to 0.95 in the placebo groups, with the SMD $(95 \% \mathrm{CI})=$ 
-0.38 [ $-0.71,-0.05]$. At the dose of $240 \mathrm{mg} /$ day, the changes in the SKT scores ranged from -2.225 to -1 in the GBE groups, and from -1.2 to 2.4 in the placebo groups, with the $\operatorname{SMD}(95 \% \mathrm{CI})=-0.59[-0.71,-0.48]$. These results indicate that treating with $240 \mathrm{mg} /$ day of GBE is more effective than treating with $160 \mathrm{mg} /$ day. The forest plot results show high heterogeneity $\left(p<0.00001, \mathrm{I}^{2}=97 \%\right)$ in both subgroups of daily doses below $240 \mathrm{mg}$, and of daily doses of $240 \mathrm{mg}$. Differences in the drug administration times, the methods of measurement, and individual variations were the potential reasons. The details are shown in Figure 7.

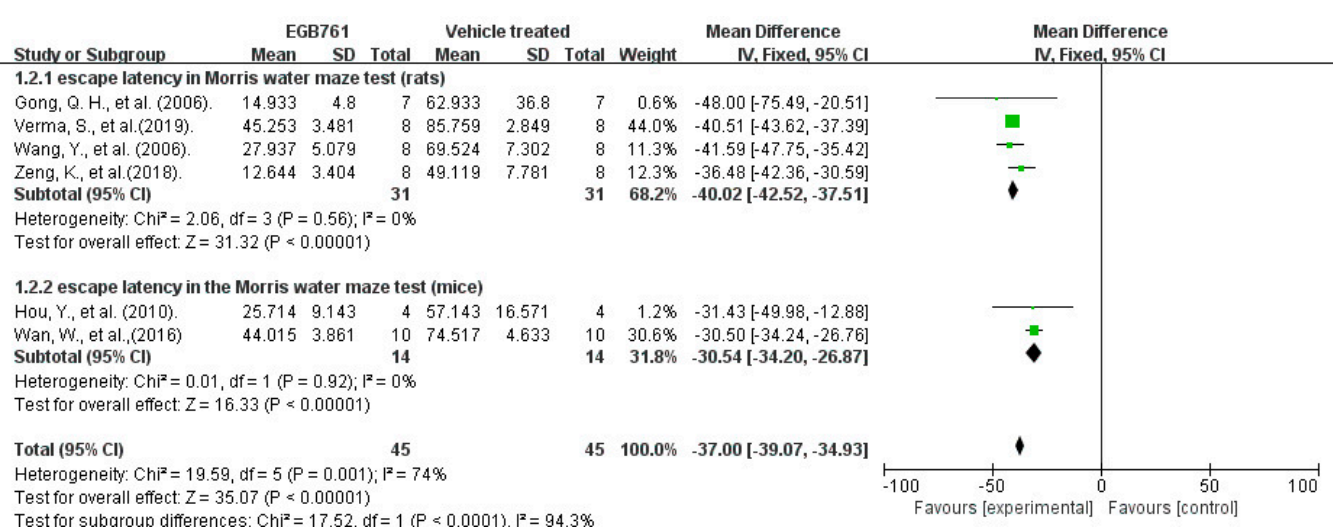

Figure 5. Forest plot for comparison: GBE treatment versus vehicle treatment. Outcome: escape latency in the probe test of MWM. The square area represents the weight assigned to the study in the meta-analysis. The horizontal line represents the $95 \%$ confidence interval.

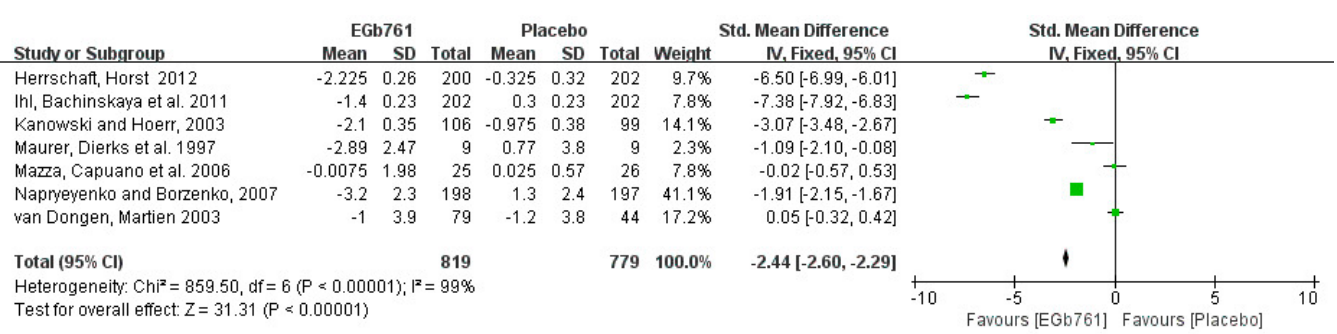

Figure 6. Meta-analysis of all patients in SKT. The square area represents the weight assigned to the study in the meta-analysis. The horizontal line represents the $95 \%$ confidence interval.

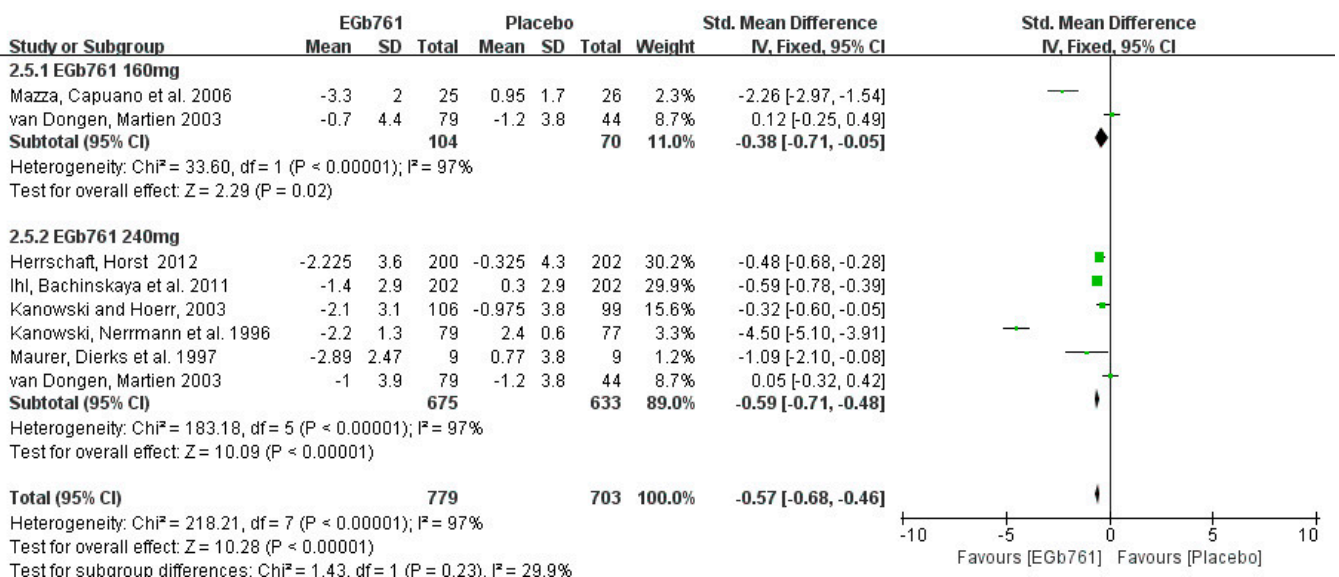

Figure 7. Forest plot of comparison: meta-analysis of AD patients taking either placebos or GBE in SKT, at doses of $240 \mathrm{mg} / \mathrm{d}$, and below $240 \mathrm{mg} / \mathrm{d}$. The square area represents the weight assigned to the study in the meta-analysis. The horizontal line represents the $95 \%$ confidence interval. 
A total of 4 of the $10(40 \%)$ effective studies evaluated the cognitive ability with ADAS-Cog. A total of 559 patients diagnosed with AD or cognitive impairment were treated with GBE (daily dose below $240 \mathrm{mg}, n=380$; daily dose of $240 \mathrm{mg}, n=179$ ), while 571 patients were treated with placebos (daily dose below $240 \mathrm{mg}, n=388$; daily dose of $240 \mathrm{mg}, n=183$ ). The univariate statistical analysis was conducted using Revman 5.3 software. The meta-analysis shows that GBE decreased the ADAS-Cog score from 31.21 to 30.33 , with the SMD $(95 \% \mathrm{CI})=-0.64[-0.78,-0.51]$. Moreover, in the forest plot of the subgroup analysis, the changes in the ADAS-Cog scores for patients taking less than a $240-\mathrm{mg}$ daily dose ranged from -0.8 to -0.2 in the GBE treatment groups, and from 1.3 to 2.8 in the placebo groups, with the SMD $(95 \% \mathrm{CI})=-1.52[-1.69,-1.35]$. At the dose of $240 \mathrm{mg} / \mathrm{d}$, the changes in the ADAS-Cog score ranged from -0.88 to -0.6 in the GBE treatment groups, and from 0.03 to 2.8 in the placebo groups, with the SMD $(95 \% \mathrm{CI})=$ $-0.59[-0.80,-0.38]$. According to these results, GBE also can decrease the ADAS-Cog scores of AD patients. The details are shown in Figure 8.

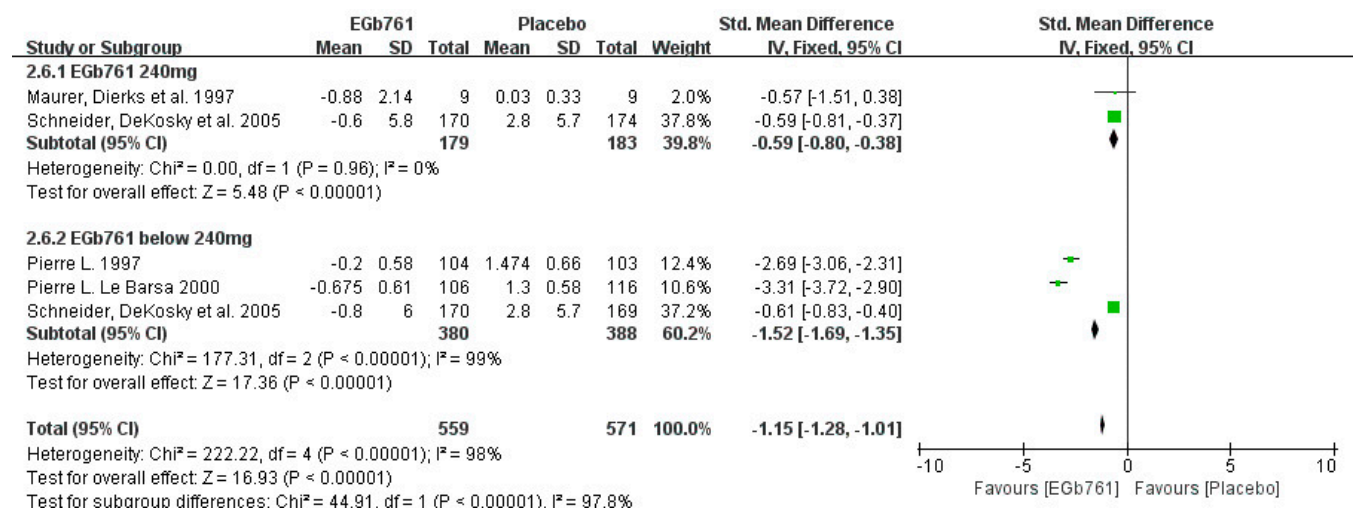

Figure 8. Forest plot of comparison: meta-analysis of ADAS-Cog scores in AD patients taking placebos or GBE, at doses of $240 \mathrm{mg} / \mathrm{d}$, and below $240 \mathrm{mg} / \mathrm{d}$. The square area represents the weight assigned to the study in the meta-analysis. The horizontal line represents the $95 \%$ confidence interval.

\subsection{Neuroprotective Mechanism Analysis}

After screening the full texts of the selected articles, we found five mechanisms that have been suggested for the neuroprotective mechanisms of GBE in AD models: reducing $\mathrm{A} \beta$ deposits and $\mathrm{p}$-Tau; anti-oxidation; antiapoptosis; anti-inflammation; and neurotransmitter balance regulation.

\subsubsection{GBE Can Significantly Reduce A $\beta$ Deposits and p-Tau in AD Models}

Previous studies have confirmed that neuronal plaques (SP), formed by amyloid- $\beta$ deposits $(A \beta)$, and neurofibrillary tangles (NFT), caused by hyperphosphorylated tau aggregation, impair the learning and memory functions of the brain (Figure 9). As a result, the inhibition of $A \beta$ aggregation and tau hyperphosphorylation have been two major strategies for alleviating AD symptoms [3].

A total of 3 of the $17(17.6 \%)$ articles detected the $A \beta$ levels in the brain tissues with immunoblotting. A total of ten TgAPP/PS1 mice were included and treated with different doses of GBE (100 mg/kg in one article, $50 \mathrm{mg} / \mathrm{kg}$ in two articles,). Another ten $\mathrm{TgAPP} / \mathrm{PS} 1$ mice were treated as a control group. The univariate statistical analysis was conducted with Revman 5.3 software. The forest plot results indicate that GBE lessened the $\mathrm{A} \beta$ deposits, both in the hippocampus and the cortex, compared with vehicle-treated animals $(p<0.00001)$, with a mean difference of 43.45 . Notably, the results of the forest plot show moderate heterogeneity $\left(p=0.11, \mathrm{I}^{2}=54 \%\right.$ ), indicating that GBE contributed to the reductions in the $A \beta$ deposits. The results of the $A \beta$ deposits were consistent with those of the behavioral test analysis. The details are shown in Figure 10. 


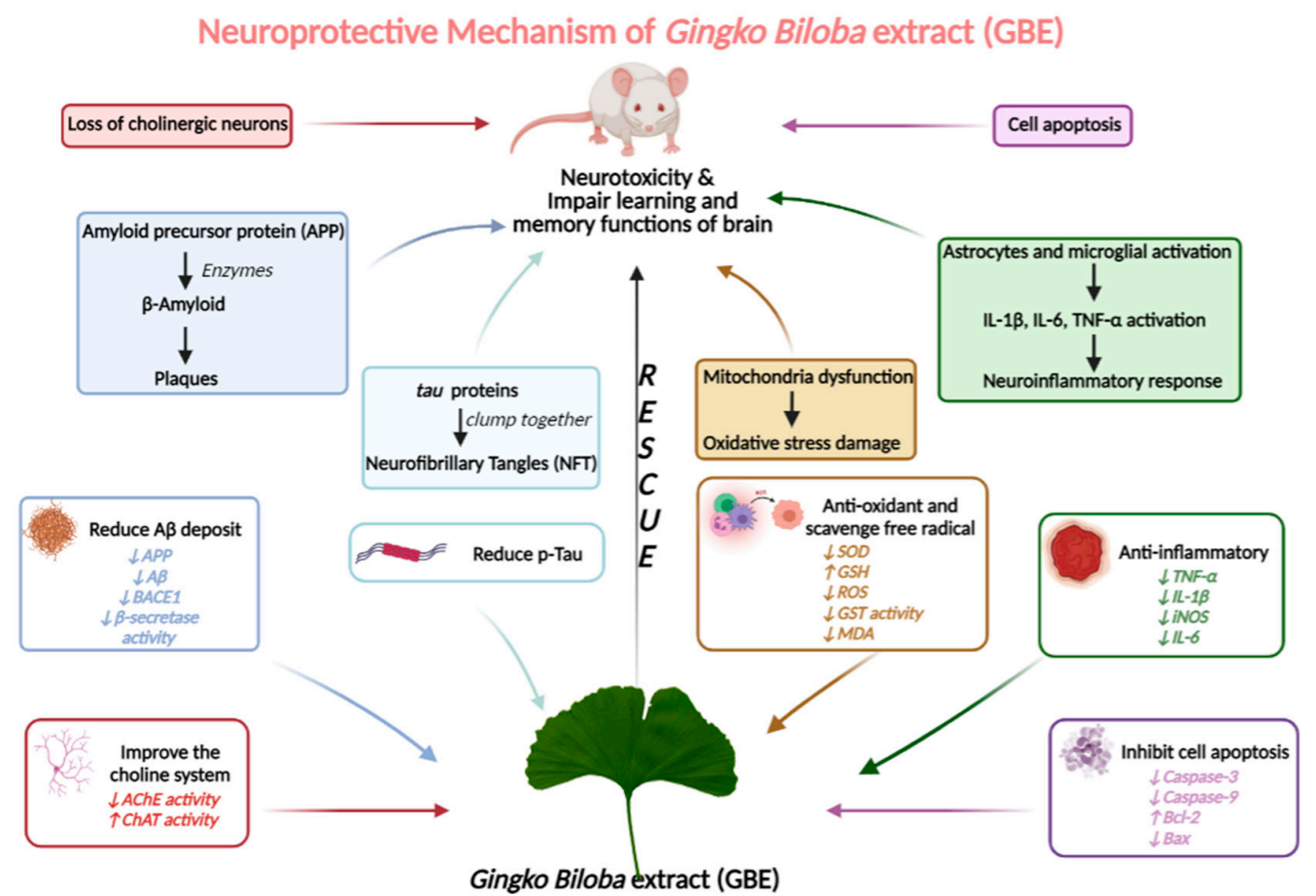

Figure 9. Possible neuroprotective mechanism of GBE.

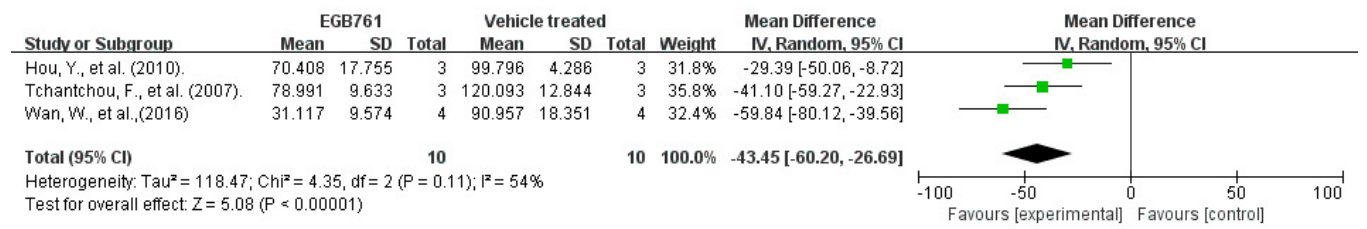

Figure 10. Forest plot for comparison: GBE treatment versus vehicle treatment. Outcome: $A \beta$ density in the brain. The square area represents the weight assigned to the study in the meta-analysis. The horizontal line represents the $95 \%$ confidence interval.

Zeng et al. (2018) [27] discovered that GBE can ameliorate the HHcy-induced tau hyperphosphorylation in the hippocampus and prefrontal cortex, and that it can further reduce the dendritic and synaptic plasticity to improve neurodegeneration. Verma et al. (2020) [29], using staining methods in an $\mathrm{AlCl}_{3}$-induced $\mathrm{AD}$ model, made it evident that GBE administration can decrease the expression of phosphorylated tau protein.

\subsubsection{GBE Displays the Antioxidant Activity}

Much research has confirmed that oxidative stress damage is a pathological feature of early AD. In the process of brain degradation, unsaturated fatty acids in the cell membranes of neurons are continuously oxidized, producing a large number of free radicals and generating peroxide lipids (LPOs). Among them, malondialdehyde (MDA) is the most toxic metabolite, and it interferes with the normal metabolism and function of neurons. The human body has free radical scavenging systems, including superoxide dismutase (SOD), glutathione peroxidase (GSH-Px), and catalase (CAT). Hence, inhibiting oxidative stress and boosting the scavenging of free radicals should be effective methods for treating $\mathrm{AD}$ (Figure 9).

Six articles have demonstrated that GBE has an antioxidative effect. Tian et al. (2012) [21] and Tian et al. (2013) [22] report that the SOD and GSH levels were significantly increased in the hippocampi of $A \beta$-injection-induced $A D$ model animals, while a significant reduction was detected in the content of MDA after GBE treatment. Protein carbonyls are often a marker of oxidative damage to proteins. Stackman et al. (2003) [26] discovered a significant increase in such carbonyls in Tg2576 mice chronically treated with GBE. In this 
case, because the GBE improved spatial learning, the increase in carbonyls may reflect a change in the brain metabolism, and not oxidative damage. Zeng et al. (2018) [27] and Verma et al. (2020) [29] report that GBE had protective effects through reducing the levels of oxygen-derived free radicals/reactive oxygen species (ROS) in hyperhomocysteinemiainduced and $\mathrm{AlCl}_{3}$-induced models. Furthermore, Verma et al. (2019) [28] confirm that GBE enhanced the GSH levels and GPx activity, while it decreased the GSSG levels and GST activity, in both the hippocampus and the cortex.

\subsubsection{GBE Inhibits Cell Apoptosis}

Multiple studies have confirmed that extensive neuronal loss is one of the major pathological features of AD, leading to learning and memory impairment in AD patients. The apoptosis mechanism of AD is closely related to: the aggregation of $A \beta$; the downregulation of apoptosis protein bcl-2 expression; the activation of the proapoptotic protein, Bax; and the activation of caspase. As a consequence, antagonistic neuron apoptosis has become an important topic in the intervention of AD. The details are shown in Figure 9.

A total of 4 of the $16(25 \%)$ articles show that GBE can inhibit cell apoptosis. The activities of caspase- 3 and caspase- 9 play important roles in cell apoptosis. Gong et al. (2005) [15] and Tian et al. (2013) [22] discovered that GBE decreases the levels of caspase-3, caspase-9, and Bax to suppress apoptosis in $\mathrm{AlCl}_{3}$-induced and $\mathrm{A} \beta$-induced $\mathrm{AD}$ models. In addition, both Jahanshahi et al. (2013) [23] and Zhang et al. (2015) [24] directly observed apoptotic cells using TUNEL staining. The images confirm that GBE protects neurons against apoptosis in the hippocampi of scopolamine-induced and $A \beta$-injection rat models.

\subsubsection{GBE Has Anti-Inflammatory Activity}

The brain's inflammatory response is an important feature of AD, and it causes a large number of neurons to undergo apoptosis in AD patients. The activation of microglial cells gives rise to the neuroinflammatory response. $A \beta$ can activate astrocytes and microglia, and can subsequently activate many inflammatory mediators, such as inflammatory cytokines (the typical inflammatory cytokines are IL-1 $\beta$, IL-6, and TNF- $\alpha$ ), nuclear transcription factors (NF-kB), and chemokines (Figure 9). These inflammatory mediators directly damage neurons in the brain and induce the excessive aggregation of $A \beta$, initiating a vicious cycle of continuous escalating damage.

In this review, five articles report that GBE had anti-inflammatory effects. Hou et al. (2010) [50] found that the oral administration of GBE increased the BDNF levels in the hippocampi of Tg APP/PS1 mice. Consistently, GBE enhanced the levels of phosphorylated CREB, which regulate the expression of BDNF in the hippocampus. Tian et al. (2012) [21] proved the protective effects of GBE by showing that the densities of damaged neurons in an A $\beta$-induced model decreased. Zhang et al. (2015) [24] reveal that GBE can activate the NF- $k B$ pathway to ameliorate the inflammatory response. Moreover, the levels of NF- $\kappa$ B-related proteins, $\mathrm{p}-\mathrm{IKK} \alpha / \beta, \mathrm{p}-\mathrm{I} \kappa \mathrm{B} \alpha$ and $\mathrm{p}-\mathrm{NF} \kappa \mathrm{B}$, were significantly increased in the A $\beta$-induced model. Liu et al. (2015) [25] discovered that GBE can markedly downregulate the transcription levels of both proinflammatory and anti-inflammatory (TNF- $\alpha$, IL- $1 \beta$, CCL-2, and IL-10), which indicated neurotoxic inflammatory inhibition in the TgCRND8 APP-transgenic mice. The evidence indicates that GBE might affect the phenotype of microglial cells. Wan et al. (2016) [26] found that treatment with GBE downregulated the proinflammatory mediators, and upregulated the anti-inflammatory cytokines, in APP/PS1 mouse brains.

\subsubsection{GBE Significantly Improves the Choline System}

The loss of cholinergic neurons is recognized as one of the major causes of dementia in the course of AD. Cholinergic neurons are severely damaged in the brains of AD patients. At the same time, choline acetyltransferase (ChAT) activity significantly decreases, while acetylcholinesterase (AChE) activity is enhanced in the brain tissue. Therefore, the level of acetylcholine is downregulated, and finally leads to dementia symptoms, including 
memory decline and cognitive dysfunction. Improving the function of the cholinergic nervous system is another strategic way to ameliorate AD. The details are shown in Figure 9.

K. Blecharz-Klin et al. (2009) [19] detected regional brain monoamine levels to find the correlation between GBE and the regulation of the neurotransmitter balance. The results show that GBE significantly increased the NA in the prefrontal cortex and hippocampus, the DOPAC in the prefrontal cortex, and the 5-HT in the striatum, all of which correlated with improved spatial memory. Increased acetylcholinesterase activity can lead to decreased acetylcholine levels, and then to reduced cognitive levels. Gong, Q. H. et al. (2006) [17], Verma et al. (2019) [28], and Verma et al. (2020) [29] discovered that AChE activity was significantly decreased in the hippocampi and cortexes of the $\mathrm{AlCl}_{3}$-induced $\mathrm{AD}$ model after GBE administration.

\subsection{Methodological Quality Analysis}

\subsubsection{Preclinical Studies}

The scores of the methodological quality for the included preclinical studies ranged from 3 to 5, out of a total of 8 points. As is shown in Table 5, all the selected articles were published in peer-reviewed journals and complied with the relevant animal welfare regulations. A total of 11 of 17 (64.7\%) [12,15-17,19,21-25,29] articles allocated animals to treatment groups or control groups randomly. Among the remaining six studies, only Stackman et al. [14] report that the behavioral tests were conducted blindly, indicating irregularities in the design of the experiments. Therefore, in order to reduce the subjective differences, which result in poor data quality, we suggest that animal experiments, especially the behavior test, should be performed in a blind manner. A total of 8 of $17(47.1 \%)[19,21,23,24,26-29]$ articles attested that they were free of potential conflicts of interest. Most of the unclaimed studies, including those of Ward et al. [12], Stackman et al. [14], Gong et al. [15], Wang et al. [16], Gong et al. [17], and Tchantchou et al. [18], were published before 2008. The lack of rigorous and complete paper-writing norms in the early stages may be responsible for this phenomenon. Only three (17.6\%) [15-17] articles assessed the dose-response relationships. Wang et al. [16] allocated Wistar rats randomly to a control group, a $30 \mathrm{mgkg}^{-1}$ EGb761 group, or a $60 \mathrm{mgkg}^{-1}$ EGb761 group, while Gong et al. $[15,17]$ divided Wistar rats randomly to a control group, a $50 \mathrm{mgkg}^{-1}$ EGb761

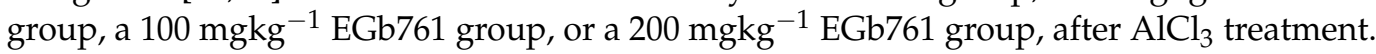
On the one hand, the samples of the animal experiment should be designed to be large enough to achieve reliable outcomes. On the other hand, animal injuries should be reduced as much as possible. A sample size calculation, to achieve sufficient power for the statistical significance, should be conducted; however, it was not performed in any of the included studies. These flaws in the methodologies of the studies obviously affect the reliability of the conclusions. All the details on the methodology qualities are shown in Table 5.

\subsubsection{Clinical Studies}

The methodological quality scores of the included clinical trials ranged from 3 to 7 , out of a total of 9 points. As is shown in Table 6, all of the selected clinical studies were published in peer-reviewed journals. Only two articles did not allocate patients to treatment groups or control groups randomly, or assess the outcomes blindly [40,41]. Amieva et al. [40] conducted an exploratory retrospective analysis of longitudinal data collected prospectively over twenty years of follow-ups of the PAQUID cohort. Similarly, Canevelli et al. [41] evaluated the effects of EGb761 in AD patients receiving cholinesterase inhibitors from a cohort study. Both of the two analyses were from cohort studies, not from randomized controlled trials. Significantly, only Schneider et al. [44] assessed the dose-response relationship of EGb761, at 120 240 mg per day. A total of 16 of 20 (80.0\%) [8,30-35,38,39,42-48] articles report the specific reasons for withdrawals during the clinical trials. Kanowski and Hoerr [36] report that a total of 222 patients were enrolled in the clinical trial, but only 205 patients were included in the analysis, with no explanation for the withdrawals. 
Mazza et al. [37] enrolled 117 AD patients and excluded 41 patients, without specifying the criteria. Canevelli et al. [41] and Amieva et al. [40] conducted cohort studies with no specific descriptions of the withdrawals. A total of 6 of 20 (30.0\%) studies conducted ITT analyses and calculated the sample sizes necessary to achieve sufficient power. One-third of the included studies had sample sizes of more than 300, and Amieva et al. [40] and Vellas et al. [47] collected more than 2000 samples. All of the details are shown in Table 6.

Table 5. Methodological qualities of GBE animal studies.

\begin{tabular}{|c|c|c|c|c|c|c|c|c|c|}
\hline \multicolumn{10}{|c|}{ Methodological Quality Scores of Included Preclinical Studies } \\
\hline & 1 & 2 & 3 & 4 & 5 & 6 & 7 & 8 & Total \\
\hline Ward, C. P. et al. (2002) [12] & $\checkmark$ & $\boldsymbol{V}$ & & & $\boldsymbol{V}$ & & $\boldsymbol{V}$ & & 4 \\
\hline Stackman, R. W. et al. (2003) [14] & $\checkmark$ & & $\checkmark$ & & $\checkmark$ & & $\boldsymbol{V}$ & & 4 \\
\hline Gong, Q. H. et al. (2005) [15] & $\checkmark$ & $\boldsymbol{V}$ & & $\checkmark$ & $\boldsymbol{V}$ & & $\boldsymbol{V}$ & & 5 \\
\hline Wang, Y. et al. (2006) [16] & $\checkmark$ & $\boldsymbol{V}$ & & $\checkmark$ & $\checkmark$ & & $\boldsymbol{V}$ & & 5 \\
\hline Gong, Q. H. et al. (2006) [17] & $\checkmark$ & $\boldsymbol{\nu}$ & & $\checkmark$ & $\boldsymbol{V}$ & & $\boldsymbol{V}$ & & 5 \\
\hline Tchantchou, F. et al. (2007) [18] & $\checkmark$ & & & & $\boldsymbol{V}$ & & $\boldsymbol{V}$ & & 3 \\
\hline Blecharz-Klin, K. et al. (2009) [19] & $\checkmark$ & $\boldsymbol{v}$ & & & $\boldsymbol{v}$ & & $\boldsymbol{V}$ & $\checkmark$ & 5 \\
\hline Hou, Y. et al. (2010) [50] & $\checkmark$ & & & & $\boldsymbol{V}$ & & $\boldsymbol{\nu}$ & & 3 \\
\hline Tian, X. et al. (2012) [21] & $\checkmark$ & $\boldsymbol{v}$ & & & $\checkmark$ & & $\checkmark$ & $\checkmark$ & 5 \\
\hline Tian, X. et al. (2013) [22] & $\checkmark$ & $\boldsymbol{V}$ & & & $\boldsymbol{V}$ & & $\boldsymbol{V}$ & & 4 \\
\hline Jahanshahi, M. et al. (2013) [23] & $\checkmark$ & $\checkmark$ & & & $\checkmark$ & & $\checkmark$ & $\checkmark$ & 5 \\
\hline Zhang, L.-D. et al. (2015) [24] & $\checkmark$ & $\boldsymbol{V}$ & & & $\checkmark$ & & $\boldsymbol{V}$ & $\checkmark$ & 5 \\
\hline Liu, X. et al. (2015) [25] & $\checkmark$ & $\boldsymbol{v}$ & & & $\checkmark$ & & $\checkmark$ & & 3 \\
\hline Wan, W. et al. (2016) [26] & $\checkmark$ & & & & $\checkmark$ & & $\boldsymbol{V}$ & $\checkmark$ & 4 \\
\hline Zeng, K. et al. (2018) [27] & $\checkmark$ & & & & $\boldsymbol{v}$ & & $\boldsymbol{V}$ & $\checkmark$ & 4 \\
\hline Verma, S. et al. (2019) [28] & $\checkmark$ & & & & $\checkmark$ & & $\checkmark$ & $\checkmark$ & 4 \\
\hline Verma, S. et al. (2020) [29] & $\checkmark$ & $\boldsymbol{v}$ & & & $\boldsymbol{V}$ & & $\boldsymbol{\nu}$ & $\checkmark$ & 5 \\
\hline
\end{tabular}

1.Was the article published in a peer-reviewed journal?

2. Were the animals allocated to the treatment group or control group randomly during the experiment?

3. Were the outcomes assessed blindly?

4. Was the dose-response relationship assessed during the experiment?

5 . Was the appropriate animal model used in the experiment?

6. Was the necessary sample size calculated to achieve sufficient power?

7. Were the animal welfare regulations complied with during the experiment?

8. Was the study free of any potential conflicts of interest?

Table 6. Methodological quality scores of GBE-related clinical trials.

\begin{tabular}{|c|c|c|c|c|c|c|c|c|c|c|}
\hline \multicolumn{11}{|c|}{ Methodological Quality Scores of Clinical Studies } \\
\hline & 1 & 2 & 3 & 4 & 5 & 6 & 7 & 8 & 9 & Total \\
\hline Schaffler and Reeh, 1985 [30] & $\boldsymbol{V}$ & $\boldsymbol{V}$ & $\boldsymbol{V}$ & & $\boldsymbol{V}$ & & & & & 4 \\
\hline Wesnes et al., 1987 [31] & $\boldsymbol{V}$ & $\boldsymbol{V}$ & $\checkmark$ & & $\boldsymbol{V}$ & & & & & 4 \\
\hline Rai et al., 1991 [32] & $\boldsymbol{V}$ & $\boldsymbol{\nu}$ & $\boldsymbol{V}$ & & $\boldsymbol{V}$ & & & & & 4 \\
\hline Kanowski, Herrmann, et al., 1996 [33] & $\boldsymbol{V}$ & $\boldsymbol{V}$ & $\boldsymbol{V}$ & & $\boldsymbol{V}$ & & & $\boldsymbol{\nu}$ & $\boldsymbol{V}$ & 6 \\
\hline Maurer, Dierks, et al., 1997 [34] & $\boldsymbol{V}$ & $\boldsymbol{\nu}$ & $\boldsymbol{\nu}$ & & $\boldsymbol{V}$ & & & & & 4 \\
\hline Barsa, Kieserc, et al., 2000 [35] & $\boldsymbol{V}$ & $\boldsymbol{v}$ & $\boldsymbol{V}$ & & $\boldsymbol{V}$ & $\boldsymbol{V}$ & $\checkmark$ & $\boldsymbol{\nu}$ & & 7 \\
\hline Kanowski and Hoerr, 2003 [36] & $\boldsymbol{V}$ & $\checkmark$ & $\boldsymbol{V}$ & & & & $\checkmark$ & & & 5 \\
\hline Mazza, Capuano, et al., 2006 [37] & $\boldsymbol{V}$ & $\checkmark$ & $\checkmark$ & & & & & & & 3 \\
\hline Napryeyenko and Borzenko, 2007 [38] & $\boldsymbol{V}$ & $\boldsymbol{V}$ & $\checkmark$ & & $\boldsymbol{V}$ & $\checkmark$ & $\checkmark$ & $\boldsymbol{V}$ & & 7 \\
\hline Ihl, Bachinskaya, et al., 2011 [39] & $\boldsymbol{V}$ & $\boldsymbol{\nu}$ & $\boldsymbol{\nu}$ & & $\boldsymbol{V}$ & & $\checkmark$ & $\boldsymbol{\nu}$ & $\checkmark$ & 7 \\
\hline Herrschaft, Nacu, et al., 2012 [8] & $\boldsymbol{V}$ & $\checkmark$ & $\checkmark$ & & $\boldsymbol{V}$ & & & $\checkmark$ & $\checkmark$ & 6 \\
\hline Amieva, Meillon, et al., 2013 [40] & $\checkmark$ & & & & & $\boldsymbol{\nu}$ & & $\boldsymbol{\nu}$ & & 3 \\
\hline Canevelli, Adali, et al., 2014 [41] & $\boldsymbol{V}$ & & & & & & & $\boldsymbol{v}$ & $\checkmark$ & 3 \\
\hline Hoerr and Nacu, 2016 [42] & $\boldsymbol{V}$ & $\checkmark$ & $\checkmark$ & & $\boldsymbol{V}$ & $\boldsymbol{V}$ & & $\checkmark$ & $\boldsymbol{V}$ & 7 \\
\hline
\end{tabular}


Table 6. Cont.

\begin{tabular}{|c|c|c|c|c|c|c|c|c|c|c|}
\hline \multicolumn{11}{|c|}{ Methodological Quality Scores of Clinical Studies } \\
\hline Subhan and Hindmarch, 1984 [43] & $\checkmark$ & $\checkmark$ & $\checkmark$ & & $\checkmark$ & & & & & 4 \\
\hline Schneider, DeKosky, et al., 2005 [44] & $\checkmark$ & $\checkmark$ & $\checkmark$ & $\checkmark$ & $\boldsymbol{\nu}$ & & $\checkmark$ & $\checkmark$ & & 7 \\
\hline McCarney, Fisher, et al., 2008 [45] & $\checkmark$ & $\checkmark$ & $\checkmark$ & & $\boldsymbol{\nu}$ & & $\checkmark$ & $\checkmark$ & $\boldsymbol{V}$ & 7 \\
\hline Snitz, O'Meara, et al., 2009 [46] & $\boldsymbol{V}$ & $\checkmark$ & $\checkmark$ & & $\boldsymbol{\nu}$ & $\checkmark$ & & $\checkmark$ & & 5 \\
\hline Vellas, Coley, et al., 2012 [47] & $\checkmark$ & $\checkmark$ & $\checkmark$ & & $\boldsymbol{\nu}$ & $\checkmark$ & & $\checkmark$ & $\checkmark$ & 7 \\
\hline Nasab, Bahrammi, et al., 2012 [48] & $\checkmark$ & $\checkmark$ & $\checkmark$ & & $\boldsymbol{V}$ & & & & & 4 \\
\hline
\end{tabular}

1.Was the article published in a peer-reviewed journal?

2.Were the patients allocated randomly during the clinical trial?

3. Were the outcomes assessed blindly?

4. Was the dose-response relationship assessed during the clinical trial?

5 . Were the withdrawals per group reported during the clinical trial?

6. Was the necessary sample size calculated to achieve sufficient power?

7. Was the ITT analysis (intent-to-treat analysis) conducted?

8. Was the funding reported for the clinical trial?

9. Was the study free of potential conflicts of interest?

\section{Discussion}

\subsection{Active Components in Gingko biloba Extract with Anti-AD Properties}

Alzheimer's disease is a brain disease with a high and increasing incidence in elderly people, and there is currently neither a cure nor an effective treatment. Currently, the drugs approved by the FDA, such as donepezil, galantamine, tacrine, and memantine, only improve the disease symptoms, without modifying the disease process, and most of them have obvious adverse effects. In general, the occurrence of $\mathrm{AD}$ is in combination with many factors, and, when some of these factors fail, new strategies arise. The amyloid hypothesis and the tau hypothesis are two mainstream explanations for the etiology of AD. However, these hypotheses are losing favor as they have failed to yield effective treatments or drugs.

The earliest use of ginkgo in China can be traced back 5000 years [58]. Today, it has been extracted to isolate a variety of constituents, including bilobalide, ginkgolides A-C, quercetin, isorhamnetin, hydroxykinurenic, rhamnose, glucose, and kaempferol. Bilobalide and ginkgolides A-C are terpenoids. They specifically inhibit platelet-activating factor (PAF) receptors [59], and are considered to be the most promising natural PAF receptor antagonists in clinical application. It is worth noting that ginkgolide $B$ can attenuate the neurotoxicity induced by $\beta$-amyloid $[10,60,61]$. Quercetin, isorhamnetin, and kaempferol are flavonoids [62]. Flavonoids are widely reported for their anti-free-radical and antioxidant effects. In addition, flavonoids have good effects in preventing and treating cardiovascular diseases, such as preventing arteriosclerosis, lowering blood lipid and cholesterol, lowering blood sugar, dilating blood vessels, improving vascular permeability, and reducing the incidence of coronary heart disease [63-65]. The administration of flavonoids could be a particularly good strategy for the prevention and treatment of the vascular symptoms related to $\mathrm{AD}$.

\subsection{Article Characteristics}

The choice of an animal model is crucial to the determination of the value of the experimental results obtained. Of the 17 included articles, 9 (52.9\%) selected the toxininduced AD model. They have the common feature of reflecting cognitive impairment. However, acute-toxicity-induced models cannot simulate the process of neurodegeneration, and these models lack NFT formation and A $\beta$ deposition. Currently, transgenic technology is the most advanced and promising measure for establishing AD models. However, only eight of the studies (out of 17, 47\%) reviewed here used transgenic-mice AD models (APP/PS1, CRND8, Tg2576) to evaluate the anti-AD effect of GBE. Their mice models showed a rapid progression of AD symptoms, but a lack of tau pathology. Future studies, 
using more widely accepted transgenic-mice AD models, such as the 3xTg and 5xFAD strains, would be useful for the further evaluation of the therapeutic effects of GBE on AD.

Although the collated data from the clinical studies assessed in this review and the methodologies of the studies were not ideal, the data seem to indicate that ginkgo biloba extract has some beneficial effects in the treatment of AD. In the clinical trials, we discovered that patients over 70 showed a low efficacy of GBE in AD treatment, while younger $\mathrm{AD}$ patients had effectively improved cognition after GBE treatment (Figure 3B). We speculate that, in the late stages of $\mathrm{AD}$, the brain has generated irreversible lesions, making it difficult to alleviate the condition [66]. This also suggests that early diagnosis and intervention are imperative in order to delay the occurrence and development of AD. Shen et al. [67] found that the detection of $\beta$-secretase (BACE1) activity in the blood may predict the onset and progression of $\mathrm{AD}$ in the early clinical stage of mild cognitive impairment (MCI). Applying this insight to clinics can reduce the medical burden on families and society. In addition, a common flaw in the clinical trials is the lack of AD hallmark evaluations. Future clinical trials on GBE would be more convincing if the AD hallmarks were included as the key indicators of disease progression.

\subsection{Future Perspective of GBE}

\subsubsection{Nanomedicine Application}

GBE is normally administered over a relatively long period (a few months) in animal models. Long-term (6 months), but not short-term (less than 6 months), GBE administration showed partial improvements in several AD clinical trials. A possible explanation is that GBE cannot cross the blood-brain barrier with high efficiency, which thereby limits its efficacy in improving cognition [68]. To solve this problem, Wang et al. [69] designed ginkgo- and corn-starch-based nanocarriers, according to the biocompatibility between ginkgo biloba extracts and starch, and they then loaded the GBE onto starch nanospheres (SNPs). Han et al. [70] designed a new system to achieve a synchronized and continuous release of EGB on the basis of an mPEG-PLGA-mPEG (PELGE) platform. Enhancing the brain penetration of GBE by pharmaceutical technique may be a feasible way to improve the efficacy of GBE.

\subsubsection{Drug Combination}

The combination treatment of GBE and donepezil significantly decreased choline levels in aged rats [71], which were consistent with the clinical trial results [37]. Canevelli et al. [41] found that GBE provides some additional cognition improvements in AD patients already under ChEIs treatment. These data indicate that the combination of GBE with classic anti-AD drugs, including ChEIs, maybe be a way to improve AD treatment efficacy.

\subsubsection{Application in Other Neurodegenerative Diseases}

Previous clinical trials have identified that the long-term administration of GBE is safe at doses of up to $240 \mathrm{mg} /$ day $[8,39,72,73]$. Multiple preclinical studies and clinical trials also reveal the neuroprotective potential of GBE. It is reasonable to speculate that GBE may also exert therapeutic activity on other neurodegenerative diseases, including Parkinson's disease. Indeed, several preclinical studies have revealed the potential protective effect of GBE on experimental PD models [74-77]. The neuroprotective activity of GBE, and the underlying mechanisms, deserve extensive investigation.

\subsubsection{Comparison of GBE Effects in Rodents and in Humans}

By comparing the meta-analysis data from human clinical trials and rodent models, we can see that GBE generally displayed more consistent and striking activity in the rodent models. In the Morris water maze experiment, GBE significantly increased the numbers of times the animals crossed the target quadrant and decreased the escape latencies, when compared with vehicle-treated animals $(p<0.00001)$, for both mice and rats. However, the SKT and ADAS-Cog scores only decreased to a certain degree after GBE treatment, with 
the SMD $(95 \% \mathrm{CI})=-2.44[-2.60,-2.29]$ (score change of SKT), and the SMD $(95 \% \mathrm{CI})=$ $-0.57[-0.68,-0.46]$ (score change of ADAS-Cog). We propose several possible reasons for this difference. First, rodents have much shorter life cycles than humans, and the duration of the GBE administration takes a larger proportion of the life cycles of mice than of humans. Second, the normalized GBE administration concentration is much higher in rodents than in humans. Third, experimental mice are inbred animals with homogenized genetic backgrounds, and the individual differences are much smaller in rodents than in humans. Fourth, the cognitive function measurements in rodent models are objective experiments, whereas, in humans, they are assessed on more subjective assessment scales.

\subsection{Conclusions}

Through the meta-analysis of preclinical studies, we find that GBE displayed predominantly positive anti-AD properties in animal models, by multiple mechanisms. Our analyses also suggest that a high dose ( $240 \mathrm{mg} /$ day) and a prolonged (over 24 weeks) administration of GBE in the early stage of AD may support improved cognitive function. However, these results should be viewed with caution given the noted methodological concerns with regard to the reviewed publications. Considering the consistent safety determinations of long-term GBE administration, future clinical trials focusing on early-stage AD patients, or on a healthy aging population with long-term GBE administration (over 24 weeks) at a high dosage (>240 mg/day), may be helpful in determining the efficacy of $\mathrm{GBE}$ in the alleviation or prevention of AD.

Author Contributions: Investigation, disposal data, writing-original draft, L.X.; methodology, writing-reviewing and editing, Q.Z.; conceptualization, funding acquisition, supervision, J.L. All authors have read and agreed to the published version of the manuscript.

Funding: This work was supported by a grant from the Natural Science Foundation of China (Grant No.: NSFC-31871024), a Grants of Macau SAR (File No. FDCT-024-2017-AMJ, FDCT-0128/2019/A3), and a University of Macau grant, MYRG2019-00129-ICMS, awarded to Jiahong Lu.

Institutional Review Board Statement: Not applicable.

Informed Consent Statement: Not applicable.

Data Availability Statement: Not applicable.

Acknowledgments: The authors would like to thank Martha Dahlen for her English editing of this manuscript.

Conflicts of Interest: The authors declare no conflict of interest.

\section{References}

1. Khachaturian, Z.S. Alzheimer's \& Dementia: The Journal of the Alzheimer's Association. Alzheimer's Dement. $2008,4,315$. [CrossRef]

2. Alzheimer's Disease International. World Alzheimer Report 2019: Attitudes to Dementia; Alzheimer's Disease International: London, UK, 2019.

3. Guillozet, A.L.; Weintraub, S.; Mash, D.C.; Mesulam, M.M. Neurofibrillary Tangles, Amyloid, and Memory in Aging and Mild Cognitive Impairment. Arch. Neurol. 2003, 60, 729-736. [CrossRef] [PubMed]

4. AsI, M. Researching Alzheimer's Medicines: Setbacks and Stepping Stones Summer. PhMRA 2015, 2015, 1-20.

5. Seo, D.-O.; Boros, B.D.; Holtzman, D.M. The microbiome: A target for Alzheimer disease? Cell Res. 2019, 29, 779-780. [CrossRef] [PubMed]

6. Drieu, K.; Jaggy, H. History, Development and Constituents of EGb 761; Harwood Academic Publishers: Amsterdam, The Netherlands, 2000.

7. $\quad$ EGb 761. Drugs R D 2003, 4, 188-193. [CrossRef]

8. Herrschaft, H.; Nacu, A.; Likhachev, S.; Sholomov, I.; Hoerr, R.; Schlaefke, S. Ginkgo biloba extract EGb $761^{\circledR}$ in dementia with neuropsychiatric features: A randomised, placebo-controlled trial to confirm the efficacy and safety of a daily dose of $240 \mathrm{mg}$. J. Psychiatr. Res. 2012, 46, 716-723. [CrossRef] [PubMed]

9. Smith, P.F.; Maclennan, K.; Darlington, C.L. The neuroprotective properties of the Ginkgo biloba leaf: A review of the possible relationship to platelet-activating factor (PAF). J. Ethnopharmacol. 1996, 50, 131-139. [CrossRef] 
10. Shi, C.; Zhao, L.; Zhu, B.; Li, Q.; Yew, D.T.; Yao, Z.; Xu, J. Protective effects of Ginkgo biloba extract (EGb761) and its constituents quercetin and ginkgolide B against $\beta$-amyloid peptide-induced toxicity in SH-SY5Y cells. Chem. Interact. 2009, 181, 115-123. [CrossRef]

11. Singh, S.K.; Srivastav, S.; Castellani, R.J.; Plascencia-Villa, G.; Perry, G. Neuroprotective and Antioxidant Effect of Ginkgo biloba Extract Against AD and Other Neurological Disorders. Neurotherapeutics 2019, 16, 666-674. [CrossRef]

12. Ward, C.P.; Redd, K.; Williams, B.M.; Caler, J.R.; Luo, Y.; McCoy, J.G. Ginkgo biloba extract: Cognitive enhancer or antistress buffer. Pharmacol. Biochem. Behav. 2002, 72, 913-922. [CrossRef]

13. Chen, J.-Y.; Zhu, Q.; Zhang, S.; Ouyang, D.; Lu, J.-H. Resveratrol in experimental Alzheimer's disease models: A systematic review of preclinical studies. Pharmacol. Res. 2019, 150, 104476. [CrossRef] [PubMed]

14. Stackman, R.W.; Eckenstein, F.; Frei, B.; Kulhanek, D.; Nowlin, J.; Quinn, J.F. Prevention of age-related spatial memory deficits in a transgenic mouse model of Alzheimer's disease by chronic Ginkgo biloba treatment. Exp. Neurol. 2003, 184, 510-520. [CrossRef]

15. Gong, Q.-H.; Wu, Q.; Huang, X.-N.; Sun, A.-S.; Shi, J.-S. Protective effects of Ginkgo biloba leaf extract on aluminum-induced brain dysfunction in rats. Life Sci. 2005, 77, 140-148. [CrossRef] [PubMed]

16. Wang, Y.; Wang, L.; Wu, J.; Cai, J. The in vivo synaptic plasticity mechanism of EGb 761-induced enhancement of spatial learning and memory in aged rats. J. Cereb. Blood Flow Metab. 2006, 148, 147-153. [CrossRef]

17. Qi-Hai, G.; Qin, W.; Xie-Nan, H.; An-Sheng, S.; Jing, N.; Jing-Shan, S. Protective effect of Ginkgo biloba leaf extract on learning and memory deficit induced by aluminum in model rats. Chin. J. Integr. Med. 2006, 12, 37-41. [CrossRef]

18. Tchantchou, F.; Xu, Y.; Wu, Y.; Christen, Y.; Luo, Y. EGb 761 enhances adult hippocampal neurogenesis and phosphorylation of CREB in transgenic mouse model of Alzheimer's disease. FASEB J. 2007, 21, 2400-2408. [CrossRef]

19. Blecharz-Klin, K.; Piechal, A.; Joniec, I.; Pyrzanowska, J.; Widy-Tyszkiewicz, E. Pharmacological and biochemical effects of Ginkgo biloba extract on learning, memory consolidation and motor activity in old rats. Acta Neurobiol. Exp. 2009, 69, $217-231$.

20. Flaks, M.K.; Forlenza, O.V.; Pereira, F.S.; Viola, L.F.; Yassuda, M.S. Short Cognitive Performance Test: Diagnostic Accuracy and Education Bias in Older Brazilian Adults. Arch. Clin. Neuropsychol. 2009, 24, 301-306. [CrossRef]

21. Tian, X.; Wang, J.; Dai, J.; Yang, L.; Zhang, L.; Shen, S.; Huang, P. Hyperbaric Oxygen and Ginkgo Biloba Extract Inhibit A $\beta 25-$ 35-induced Toxicity and Oxidative Stress in vivo: A Potential Role in Alzheimer's Disease. Int. J. Neurosci. 2012, 122, 563-569. [CrossRef]

22. Tian, X.; Zhang, L.; Wang, J.; Dai, J.; Shen, S.; Yang, L.; Huang, P. The protective effect of hyperbaric oxygen and Ginkgo biloba extract on A $\beta 25-35$-induced oxidative stress and neuronal apoptosis in rats. Behav. Brain Res. 2013, 242, 1-8. [CrossRef]

23. Jahanshahi, M.; Nickmahzar, E.G.; Babakordi, F. The effect of Ginkgo biloba extract on scopolamine-induced apoptosis in the hippocampus of rats. Anat. Sci. Int. 2013, 88, 217-222. [CrossRef] [PubMed]

24. Zhang, L.-D.; Ma, L.; Dai, J.-G.; Chang, L.-G.; Huang, P.-L.; Tian, X.-Q. Hyperbaric Oxygen and Ginkgo Biloba Extract Ameliorate Cognitive and Memory Impairment via Nuclear Factor Kappa-B Pathway in Rat Model of Alzheimer's Disease. Chin. Med. J. 2015, 128, 3088-3093. [CrossRef] [PubMed]

25. Liu, X.; Hao, W.; Qin, Y.; Decker, Y.; Wang, X.; Burkart, M.; Schötz, K.; Menger, M.D.; Fassbender, K.; Liu, Y. Long-term treatment with Ginkgo biloba extract EGb 761 improves symptoms and pathology in a transgenic mouse model of Alzheimer's disease. Brain Behav. Immun. 2015, 46, 121-131. [CrossRef] [PubMed]

26. Wan, W.; Zhang, C.; Danielsen, M.; Li, Q.; Chen, W.; Chan, Y.; Li, Y. EGb761 improves cognitive function and regulates inflammatory responses in the APP/PS1 mouse. Exp. Gerontol. 2016, 81, 92-100. [CrossRef] [PubMed]

27. Zeng, K.; Li, M.; Xinghua, L.; Mahaman, Y.A.R.; Bao, J.; Huang, F.; Xiaochuan, W.; Liu, X.; Wang, Q.; Wang, J.-Z.; et al Ginkgo biloba Extract EGb761 Attenuates Hyperhomocysteinemia-induced AD Like Tau Hyperphosphorylation and Cognitive Impairment in Rats. Curr. Alzheimer Res. 2017, 15, 89-99. [CrossRef] [PubMed]

28. Verma, S.; Ranawat, P.; Sharma, N.; Nehru, B. Ginkgo biloba attenuates aluminum lactate-induced neurotoxicity in reproductive senescent female rats: Behavioral, biochemical, and histopathological study. Environ. Sci. Pollut. Res. 2019, 26, $27148-27167$. [CrossRef] [PubMed]

29. Verma, S.; Sharma, S.; Ranawat, P.; Nehru, B. Modulatory Effects of Ginkgo biloba Against Amyloid Aggregation Through Induction of Heat Shock Proteins in Aluminium Induced Neurotoxicity. Neurochem. Res. 2020, 45, 465-490. [CrossRef]

30. Schaffler, K.; Reeh, P. Double blind study of the hypoxia protective effect of a standardized Ginkgo biloba preparation after repeated administration in healthy subjects. Arzneimittelforschung 1985, 35, 1283-1286.

31. Wesnes, K.; Simmons, D.; Rook, M.; Simpson, P. A double-blind placebo-controlled trial of tanakan in the treatment of idiopathic cognitive impairment in the elderly. Hum. Psychopharmacol. Clin. Exp. 1987, 2, 159-169. [CrossRef]

32. Rai, G.S.; Shovlin, C.; Wesnes, K.A. A double-blind, placebo controlled study of Ginkgo biloba extract ('Tanakan') in elderly outpatients with mild to moderate memory impairment. Curr. Med. Res. Opin. 1991, 12, 350-355. [CrossRef]

33. Kanowski, S.; Herrmann, W.; Stephan, K.; Wierich, W.; Hörr, R. Proof of Efficacy of the Ginkgo Biloba Special Extract EGb 761 in Outpatients Suffering from Mild to Moderate Primary Degenerative Dementia of the Alzheimer Type or Multi-infarct Dementia. Pharmacopsychiatry 1996, 29, 47-56. [CrossRef] [PubMed]

34. Maurer, K.; Ihl, R.; Dierks, T.; Frölich, L. Clinical efficacy of Ginkgo biloba special extract EGb 761 in dementia of the Alzheimer type. J. Psychiatr. Res. 1997, 31, 645-655. [CrossRef]

35. BBarsa, P.L.L.; Kieserc, M.; Itilb, K.Z. A 26-week analysis of a double-blind, placebo-controlled trial of the ginkgo biloba extract EGb $761^{\circledR}$ in Dementia. Dement. Geriatr. Cogn. Disord. 2000, 11, 230-237. [CrossRef] [PubMed] 
36. Kanowski, S.; Hoerr, R. Ginkgo biloba Extract EGb $761{ }^{\circledR}$ in Dementia: Intent-to-treat Analyses of a 24-week, Multi-center, Double-blind, Placebo-controlled, Randomized Trial. Pharmacopsychiatry 2003, 36, 297-303. [CrossRef]

37. Mazza, M.; Capuano, A.; Bria, P.; Mazza, S. Ginkgo biloba and donepezil: A comparison in the treatment of Alzheimer's dementia in a randomized placebo-controlled double-blind study. Eur. J. Neurol. 2006, 13, 981-985. [CrossRef] [PubMed]

38. Napryeyenko, O.; Borzenko, I. Ginkgo biloba Special Extract in Dementia with Neuropsychiatric Features. Arzneimittelforschung 2007, 57, 4-11. [CrossRef] [PubMed]

39. Ihl, R.; Bachinskaya, N.; Korczyn, A.D.; Vakhapova, V.; Tribanek, M.; Hoerr, R.; Napryeyenko, O. Efficacy and safety of a once-daily formulation of Ginkgo biloba extract EGb 761 in dementia with neuropsychiatric features: A randomized controlled trial. Int. J. Geriatr. Psychiatry 2010, 26, 1186-1194. [CrossRef]

40. Amieva, H.; Meillon, C.; Helmer, C.; Barberger-Gateau, P.; Dartigues, J.F. Ginkgo Biloba Extract and Long-Term Cognitive Decline: A 20-Year Follow-Up Population-Based Study. PLoS ONE 2013, 8, e52755. [CrossRef]

41. Canevelli, M.; Adali, N.; Kelaiditi, E.; Cantet, C.; Ousset, P.-J.; Cesari, M. Effects of Gingko biloba supplementation in Alzheimer's disease patients receiving cholinesterase inhibitors: Data from the ICTUS study. Phytomedicine 2014, 21, 888-892. [CrossRef]

42. Hoerr, R.; Nacu, A. Neuropsychiatric symptoms in dementia and the effects of Ginkgo biloba extract EGb $761^{\circledR}$ treatment: Additional results from a 24-week randomized, placebo-controlled trial. Open Access J. Clin. Trials 2016, 8, 1-6. [CrossRef]

43. Subhan, Z.; Hindmarch, I. The psychopharmacological effects of Ginkgo biloba extract in normal healthy volunteers. Int. J. Clin. Pharmacol. Res. 1984, 4, 89-93. [PubMed]

44. Lon, S.; Schneider, S.T.; DeKosky, M.R.; Farlow, P.N.; Tariot, H.R.; Kieser, M. A randomized, double-blind, placebo-controlled trial of two doses of ginkgo biloba extract in dementia of the Alzheimer's type. Curr. Alzheimer Res. 2005, 2, 541-551.

45. McCarney, R.; Fisher, P.; Iliffe, S.; van Haselen, R.; Griffin, M.; Van Der Meulen, J.; Warner, J. Ginkgo biloba for mild to moderate dementia in a community setting: A pragmatic, randomised, parallel-group, double-blind, placebo-controlled trial. Int. J. Geriatr. Psychiatry 2008, 23, 1222-1230. [CrossRef] [PubMed]

46. Snitz, B.E.; O’Meara, E.S.; Carlson, M.C.; Arnold, A.M.; Ives, D.G.; Rapp, S.R.; Saxton, J.; Lopez, O.L.; Dunn, L.O.; Sink, K.; et al. Ginkgo biloba for Preventing Cognitive Decline in Older AdultsA Randomized Trial. JAMA J. Am. Med. Assoc. 2009, 302, 2663-2670. [CrossRef]

47. Vellas, B.; Coley, N.; Ousset, P.-J.; Berrut, G.; Dartigues, J.-F.; Dubois, B.; Grandjean, H.; Pasquier, F.; Piette, F.; Robert, P.; et al. Long-term use of standardised ginkgo biloba extract for the prevention of Alzheimer's disease (GuidAge): A randomised placebo-controlled trial. Lancet Neurol. 2012, 11, 851-859. [CrossRef]

48. Nasab, N.M.; Bahrammi, M.A.; Nikpour, M.R.A.; Rahim, F.; Naghibis, S.N. Efficacy of rivastigmine in comparison to ginkgo for treating Alzheimer's dementia. J. Pak. Med. Assoc. 2012, 62, 677-680.

49. Zhu, Q.; Zhuang, X.; Lu, J. Neuroprotective effects of baicalein in animal models of Parkinson's disease: A systematic review of experimental studies. Phytomedicine 2019, 55, 302-309. [CrossRef]

50. Hou, Y.; Aboukhatwa, M.A.; Lei, D.-L.; Manaye, K.; Khan, I.; Luo, Y. Anti-depressant natural flavonols modulate BDNF and beta amyloid in neurons and hippocampus of double TgAD mice. Neuropharmacology 2010, 58, 911-920. [CrossRef]

51. American Psychiatric Association. Diagnostic and Statistical Manual of Mental Disorders (DSM-5®); American Psychiatric Pub: Washington, DC, USA, 2013.

52. World Health Organization. The ICD-10 Classification of Mental and Behavioural Disorders: Diagnostic Criteria for Research; World Health Organization: Geneva, Switzerland, 1993.

53. Tombaugh, T.; McDowell, I.; Kristjansson, B.; Hubley, A. Mini-Mental State Examination (MMSE) and the Modified MMSE (3MS): A psychometric comparison and normative data. Psychol. Assess. 1996, 8, 48. [CrossRef]

54. Kueper, J.K.; Speechley, M.; Montero-Odasso, M. The Alzheimer's Disease Assessment Scale-Cognitive Subscale (ADAS-Cog): Modifications and Responsiveness in Pre-Dementia Populations. A Narrative Review. J. Alzheimer's Dis. 2018, 63, 423-444. [CrossRef]

55. Dubois, B.; Feldman, H.H.; Jacova, C.; DeKosky, S.T.; Barberger-Gateau, P.; Cummings, J.; Delacourte, A.; Galasko, D.; Gauthier, S.; Jicha, G. Research criteria for the diagnosis of Alzheimer's disease: Revising the NINCDS-ADRDA criteria. Lancet Neurol. 2007, 6, 734-746. [CrossRef]

56. Paul, R.; Solomon, P.; Felicity Adams, B.; Amanda Silver, B.; Jill Zimmer, B.; Richard DeVeaux, P. Ginkgo for Memory Enhancement: A Randomized Controlled Trial; American Medical Association: Chicago, IL, USA, 2002.

57. Schoenfeld, R.; Schiffelholz, T.; Beyer, C.; Leplow, B.; Foreman, N. Variants of the Morris water maze task to comparatively assess human and rodent place navigation. Neurobiol. Learn. Mem. 2017, 139, 117-127. [CrossRef]

58. Griggs, B. The History and Evolution of Western Herbal Medicine; Healing Arts Press: London, UK; Rochester, VT, USA, 1997; p. 448.

59. Yue, T.L.; Feuerstein, G.Z. Platelet-activating factor: A putative neuromodulator and mediator in the pathophysiology of brain injury. Crit. Rev. Neurobiol. 1994, 8, 11-24. [PubMed]

60. Kaur, N.; Dhiman, M.; Perez-Polo, J.R.; Mantha, A.K. Ginkgolide B revamps neuroprotective role of apurinic/apyrimidinic endonuclease 1 and mitochondrial oxidative phosphorylation against A $\beta 25$-35-induced neurotoxicity in human neuroblastoma cells. J. Neurosci. Res. 2015, 93, 938-947. [CrossRef] [PubMed]

61. Gill, I.; Kaur, S.; Kaur, N.; Dhiman, M.; Mantha, A.K. Phytochemical Ginkgolide B Attenuates Amyloid- $\beta 1-42$ Induced Oxidative Damage and Altered Cellular Responses in Human Neuroblastoma SH-SY5Y Cells. J. Alzheimer's Dis. 2017, 60, S25-S40. [CrossRef] [PubMed] 
62. Hämäläinen, M.; Nieminen, R.; Vuorela, P.; Heinonen, M.; Moilanen, E. Anti-inflammatory effects of flavonoids: Genistein, kaempferol, quercetin, and daidzein inhibit STAT-1 and NF- $\mathrm{kB}$ activations, whereas flavone, isorhamnetin, naringenin, and pelargonidin inhibit only NF- $\mathrm{B}$ activation along with their inhibitory effect on iNOS expression and NO production in activated macrophages. Mediat. Inflamm. 2007, 2007, 45673. [CrossRef]

63. Gohil, K.; Moy, R.K.; Farzin, S.; Maguire, J.J.; Packer, L. mRNA expression profile of a human cancer cell line in response to Ginkgo biloba extract: Induction of antioxidant response and the Golgi system. Free Radic. Res. 2000, 33, 831-849. [CrossRef]

64. Dower, J.I.; Geleijnse, J.M.; Gijsbers, L.; Schalkwijk, C.; Kromhout, D.; Hollman, P.C. Supplementation of the Pure Flavonoids Epicatechin and Quercetin Affects Some Biomarkers of Endothelial Dysfunction and Inflammation in (Pre)Hypertensive Adults: A Randomized Double-Blind, Placebo-Controlled, Crossover Trial. J. Nutr. 2015, 145, 1459-1463. [CrossRef]

65. Dower, J.I.; Geleijnse, J.M.; Gijsbers, L.; Zock, P.; Kromhout, D.; Hollman, P.C.H. Effects of the pure flavonoids epicatechin and quercetin on vascular function and cardiometabolic health: A randomized, double-blind, placebo-controlled, crossover trial. Am. J. Clin. Nutr. 2015, 101, 914-921. [CrossRef]

66. Alzheimer's disease: Recent progress and prospects-Part III. Harv. Ment. Health Lett. 2001, 18, 1-4.

67. Shen, Y.; Wang, H.; Sun, Q.; Yao, H.; Keegan, A.P.; Mullan, M.; Wilson, J.; Lista, S.; Leyhe, T.; Laske, C.; et al. Increased Plasma Beta-Secretase 1 May Predict Conversion to Alzheimer's Disease Dementia in Individuals With Mild Cognitive Impairment. Biol. Psychiatry 2018, 83, 447-455. [CrossRef] [PubMed]

68. Di Martino, P.; Censi, R.; Gigliobianco, M.R.; Zerrillo, L.; Magnoni, F.; Agas, D.; Quaglia, W.; Lupidi, G. Nano-medicine Improving the Bioavailability of Small Molecules for the Prevention of Neurodegenerative Diseases. Curr. Pharm. Des. 2017, 23, 1897-1908. [CrossRef] [PubMed]

69. Wang, T.; Wu, C.; Fan, G.; Li, T.; Gong, H.; Cao, F. Ginkgo biloba extracts-loaded starch nano-spheres: Preparation, characterization, and in vitro release kinetics. Int. J. Biol. Macromol. 2018, 106, 148-157. [CrossRef] [PubMed]

70. Han, L.; Fu, Y.; Cole, A.J.; Liu, J.; Wang, J. Co-encapsulation and sustained-release of four components in ginkgo terpenes from injectable PELGE nanoparticles. Fitoterapia 2012, 83, 721-731. [CrossRef] [PubMed]

71. Stein, C.; Hopfeld, J.; Lau, H.; Klein, J. Effects of Ginkgo biloba Extract EGb 761, Donepezil and their Combination on Central Cholinergic Function in Aged Rats. J. Pharm. Pharm. Sci. 2015, 18, 634-646. [CrossRef]

72. Hashiguchi, M.; Ohta, Y.; Shimizu, M.; Maruyama, J.; Mochizuki, M. Meta-analysis of the efficacy and safety of Ginkgo biloba extract for the treatment of dementia. J. Pharm. Health Care Sci. 2015, 1, 1-12. [CrossRef]

73. Ihl, R. Effects of Ginkgo biloba extract EGb $761^{\circledR}$ in dementia with neuropsychiatric features: Review of recently completed randomised, controlled trials. Int. J. Psychiatry Clin. Pract. 2013, 17, 8-14. [CrossRef]

74. Rojas, P.; Montes, S.; Serrano-García, N.; Rojas-Castañeda, J. Effect of EGb761 supplementation on the content of copper in mouse brain in an animal model of Parkinson's disease. Nutrition 2009, 25, 482-485. [CrossRef]

75. Rojas, P.; Serrano-García, N.; Mares-Sámano, J.J.; Medina-Campos, O.N.; Pedraza-Chaverri, J.; Ögren, S.O. EGb761 protects against nigrostriatal dopaminergic neurotoxicity in 1-methyl-4-phenyl-1,2,3,6-tetrahydropyridine-induced Parkinsonism in mice: Role of oxidative stress. Eur. J. Neurosci. 2008, 28, 41-50. [CrossRef]

76. Ahmad, M.; Saleem, S.; Ahmad, A.S.; Yousuf, S.; Ansari, M.A.; Khan, M.B.; Ishrat, T.; Chaturvedi, R.K.; Agrawal, A.K.; Islam, F. Ginkgo biloba affords dose-dependent protection against 6-hydroxydopamine-induced parkinsonism in rats: Neurobehavioural, neurochemical and immunohistochemical evidences. J. Neurochem. 2005, 93, 94-104. [CrossRef]

77. Yu, D.; Zhang, P.; Li, J.; Liu, T.; Zhang, Y.; Wang, Q.; Zhang, J.; Lu, X.; Fan, X. Neuroprotective effects of Ginkgo biloba dropping pills in Parkinson's disease. J. Pharm. Anal. 2021, 11, 220-231. [CrossRef] [PubMed] 